\title{
Les énoncés «présentatifs» chez les apprenants sinophones de français $L 2$
}

\author{
Ludovica Lena $^{1}$ \\ ${ }^{1}$ Centre de Recherches Linguistiques sur l'Asie Orientale, UMR8563, INALCO, EHESS et CNRS, \\ France et Université La Sapienza, Rome, Italie
}

\begin{abstract}
Résumé. La présente étude s'intéresse à l'organisation du discours chez les apprenants sinophones de français L2, en analysant leur maîtrise des procédés syntaxiques associés à la fonction d'introduction référentielle. Cette recherche part du constat que le français et le chinois L1 se servent de stratégies syntaxiques très proches: les francophones recourent massivement à la construction en « il y a » suivie d'une proposition relative, tandis que les sinophones disposent d'une structure présentative dont le premier segment est introduit par le verbe yǒu 'avoir'. Nos données montrent que si les apprenants sinophones utilisent les structures en AVOIR, ainsi que les autres tournures syntaxiques portant la même fonction textuelle, ils n'y recourent que dans une moindre mesure. Une analyse des caractéristiques propres à ces structures nous montre que les apprenants transposent dans la langue cible les principes qui guident la structuration de l'information dans leur L1 - notamment en ne parvenant pas à associer à la structure en AVOIR une fonction pragmatique dont est dépourvue la forme correspondante dans la langue source (leur L1); d'autre part la connaissance métalinguistique de la L2 semble jouer un rôle important dans la mesure où les apprenants rétablissent souvent l'ordre non marqué $\mathrm{S}-\mathrm{V}$, même dans des contextes pragmatiquement inappropriés.
\end{abstract}

\begin{abstract}
Acquisition of pragmatically motivated syntax by Chinese learners of L2 French. The present study focuses on the discourse organization adopted by Chinese-speaking learners of L2 French, and analyzes how they manage the syntactic patterns that introduce new referential entities into discourse. French and Chinese L1 happen to use for this purpose very similar syntactic strategies: in French the common pattern is the bi-clausal il y a 'there is' construction (from avoir 'have'), while in Chinese new referents are often encoded by a presentational structure whose first segment is introduced by the semantically-related verb yǒu 'have'. Our data show that Chinese-speaking learners do use the HAVE-presentational structures as well as the other syntactic patterns carrying the same textual function, but to a smaller extent. By analyzing the characteristics of these structures we show that Chinese speakers transpose in the target language the same principles that guide the information structuring in their L1 - in particular they fail to link the HAVE-structure to a pragmatic function lacking for the corresponding form in the source language ; secondly the L2
\end{abstract}


metalinguistic knowledge seems to play an important role in that learners often choose the unmarked S-V word-order, even in contexts that are pragmatically inappropriate.

\section{Introduction : cadre théorique et problématique}

Dans le cadre théorique des recherches d'inspiration fonctionnaliste sur l'acquisition (Klein et Perdue 1992), nous prenons comme point de départ la considération que les différences interlinguistiques peuvent avoir une incidence sur la manière dont se développe l'organisation de l'information au niveau du discours et de l'énoncé (Carroll et Lambert 2006 ; Hendriks et Watorek 2008).

De nombreuses recherches antérieures sur l'acquisition du français langue étrangère soulignent l'impact de la langue source : entre autres, l'étude de Hendriks et Watorek (2008) montre que l'organisation de l'information en topique chez les apprenants adultes se fait à travers le recours à des moyens linguistiques calqués sur ceux de leurs langues sources. Lambert et al. (2008) ont étudié l'influence de la langue maternelle dans les modes de résolution de tâches verbales complexes, en montrant que les L2 tendent à conceptualiser et organiser les contenus informatifs sur la base des principes de leur L1 : les productions des apprenants se caractérisent par des divergences systématiques dans la structuration de l'information en discours qui reflètent l'influence de la langue source, indépendamment du niveau de maîtrise linguistique. Dans son étude sur les locuteurs francophones d'anglais L2, Leclercq (2008) montre que les apprenants quasi-bilingues sont toujours influencés par la structuration informationnelle de la langue source : « les codages opérés en langue maternelle influenceraient les locuteurs lors de l'expression en L2, même à un niveau très avancé ». Spécifiquement, Lambert (1998) montre que la L1 peut influencer la manière d'introduire de nouveaux référents dans le discours.

Nous nous sommes tout particulièrement inspirée de l'étude conduite par Turco (2008) sur l'acquisition des structures syntaxiques dédiées à l'encodage des fonctions d'introduction et d'identification des référents par les apprenants francophones avancés de l'italien L2. Son travail montre que ces locuteurs utilisent aisément les structures présentatives clivées de l'italien « c'è $\mathrm{SN}^{1}$ che », lesquelles correspondent aux formes en il y a de leur langue source, tandis que l'apparition de l'ordre verbe-sujet (V-S) chez les apprenants est conditionnée en même temps par plusieurs facteurs tels que le statut des référents, les propriétés sémantiques du verbe - elle se déclenche notamment en présence de verbes inaccusatifs, et le contexte énonciatif, qui doit être de nature thétique ${ }^{2}$.

La structure informationnelle étant indissociable des moyens formels disponibles dans chaque langue, l'apprenant d'une L2 doit en effet, afin d'acquérir une compétence native, parvenir à identifier les corrélations entre une forme et sa fonction dans la langue cible pour parvenir à assurer les opérations de référenciation et organiser efficacement son discours (Carroll et Lambert 2003) : «contrairement aux enfants qui acquièrent conjointement leur langue et son usage, les apprenants doivent abandonner les principes de gestion qu'ils ont intériorisés avec et par leur langue source pour en adopter d'autres. On peut se demander si cela ne constitue pas un obstacle quasiment infranchissable » (Lambert et al. 2008).

\footnotetext{
${ }^{1}$ Syntagme nominal

${ }^{2}$ Les énoncés « thétiques » (Kuroda 1972) ou de type « all-focus » (Lambrecht 1987a) ont la fonction de présenter une entité, une proposition ou un état de choses en tant qu'élément d'information nouveau pour le discours. Ces énoncés ne présupposent qu'un contexte discursif minimal et peuvent ainsi apparaître au tout début d'un discours et répondre à la question « qu'est ce qui se passe ? » ou bien présenter un événement d'arrière-plan (Cornish 2005).
} 
Nous nous penchons sur la question de l'influence de la L1 sur l'organisation discursive de la L2 en analysant de quelle manière les locuteurs natifs et les apprenants sinophones du français L2 encodent l'introduction des nouvelles entités référentielles dans le discours. La présente étude part du constat que les deux langues examinées, le chinois et le français, s'appuient systématiquement sur des procédés syntaxiques pour exprimer la fonction d'introduction référentielle, et que toute deux recourent en particulier pour ce faire aux structures « présentatives » en AVOIR.

On pourrait penser à première vue que la présence dans la langue source d'une structure syntaxique marquée qui trouve un correspondant dans la langue cible devrait favoriser sa maîtrise de la part des apprenants, comme c'est le cas par exemple des apprenants hispanophones vis-à-vis des structures à ordre V-S en italien L2 (Chini 2001), ou des francophones lorsqu'ils produisent des structures clivées du type «c'è SN che » en italien L2 (Turco 2008). Parfois les apprenants tendent à surexploiter les structures «présentatives », en recourant davantage à des tournures analytiques pour introduire un personnage dans la narration, lorsque les natifs auraient préféré des solutions synthétiques comme l'ordre des mots ou la structure à SN seule. Ainsi, les apprenants francophones d'italien L2 utilisent la construction présentative clivée même dans des contextes où elle est redondante - notamment avec des verbes inaccusatifs. On a aussi constaté que les apprenants francophones avancés de l'anglais L2 ont tendance à s'appuyer largement sur des clivages syntaxiques, tandis que les anglophones natifs ont davantage recours à des procédés de type prosodique pour structurer l'information dans le discours. Notamment les apprenants utilisent massivement les structures présentatives pour assurer l'ancrage énonciatif, faisant ainsi appel à un mode d'ancrage explicite (Lambert 1998). Leclercq (2008) relève chez les apprenants francophones des formes non attestées dans les récits des anglophones, notamment le présentatif «I can see » ou l'emploi de subordonnées introduites par who.

Dans cette étude, nous analysons les productions d'apprenants sinophones pour observer comment ils gèrent la mise en place des structures présentatives en français L2 dans la résolution d'une tache verbale de compte-rendu de vidéo. Etant donné que leur langue maternelle présente une structure présentative en AVOIR, et que cette forme est en chinois systématiquement employée avec la même fonction d'ancrage des nouveaux référents qu'en français, la langue cible, cela pourrait faciliter son apparition dans les productions des apprenants sinophones. D'autre part, il existe des différences interlangues demandant une subtile maîtrise pragmatique en plus de la maîtrise formelle de la grammaire de la langue cible. Cette acquisition présuppose de parvenir à associer à la structure de la L2 des nouvelles fonctions que l'on ne trouve pas dans la forme correspondante en L1 ou à l'inverse de dissocier les formes des usages pragmatiques qui sont propres aux configurations utilisées en langue maternelle.

Dans son étude des apprenants sinophones peu avancés d'italien L2, Valentini recense des constructions présentatives ( «c'è SN che ») et des inversions V-S (Valentini 1992 : 113117, reporté en Chini 2002) :

(1) IT2_CH1

ha morti tante persone

'beaucoup de gens sont morts' (Valentini 1992 : 113)

En étudiant la production narrative d'une jeune locutrice sinophone en italien L2, Chini (2002) remarque que les inversions V-S sont moins fréquentes par rapport aux formules existentielles ; cependant, l'apprenante « ne présente pas de cas d'ordres SV là où l'inversion VS est nécessaire. En chinois aussi, ce type de verbe [= inaccusatif] a souvent l'inversion, surtout si son sujet est une entité nouvelle du discours ». Les exemples suivant sont adaptés de Chini (2002; ses exemples 49 et 50) : 
(2) IT2_CH1

(3) IT2_CH1 e poi cosa succede? ('et ensuite que se passe-t-il ?') poi co/pecché poi co/ ++ cadono le mele ('les pommes tombent')

e poi eh son:++

sono morti tutti in cinesi ('les chinois sont morts tous')

L'auteure estime que la langue maternelle de l'apprenante semble influente en italien L2 dans la mesure où « elle favorise la manipulation pragmatique de l'ordre des mots ». Ces études montrent que l'acquisition de l'ordre V-S présentatif chez les locuteurs sinophones serait facilitée car cette structure est déjà mise à disposition par la L1. Or étant donné que l'emploi de V-S en français oral est attesté mais n'est pas autant fréquent qu'en chinois, cela pourrait influencer également les productions des apprenants.

Ainsi, nous nous proposons d'étudier comment les apprenants sinophones avancés s'adaptent aux contraintes pragmatiques en français langue cible, étant donné que l'appropriation des principes pragmatiques est souvent évoquée comme la dernière étape avant une maîtrise réussie de la lange seconde (hypothèse avancée par Perdue 1993). Ce travail prend comme point de départ l'analyse des structures syntaxiques (formelles) qui interviennent pour encoder la fonction d'introduction référentielle (identifiée grâce au contexte fourni par le support visuel). Les questions qui guident l'étude de nos données sont donc les suivantes :

- Quels sont les moyens linguistiques encodant la fonction d'introduction référentielle en chinois et en français L1 et notamment par quels marqueurs syntaxiques se manifesteelle?

- La présence dans la langue source d'un même type de structure "présentative » (en particulier les clivées en AVOIR et l'ordre V-S) favorise-t-elle son emploi de la part des apprenants sinophones en français langue cible?

- Comment les apprenants sinophones s'approprient-ils des différentes contraintes d'ordre pragmatique propres aux structures « présentatives » de la langue cible ?

L'article est ainsi structuré : on présentera d'abord la méthodologie adoptée (section 2), puis nous allons conduire une comparaison des procédés syntaxiques déployés pour introduire les référents dans le discours narratif en chinois et en français L1 (section 3), et enfin nous analysons les stratégies employées par les apprenants sinophones de français L2 (section 4).

\section{Méthodologie}

Sur la base d'une tâche de récit en langue seconde d'un film muet de la part de locuteurs sinophones, nous avons recueilli deux corpora de chinois L1 et français L2, pour conduire une analyse croisée avec les narrations collectées en français L1.

Le support employé pour recueillir les données est un montage tiré du film muet Les Temps modernes de Charlie Chaplin, élaboré dans le cadre du projet ESF (European Science Foundation ; cf. Klein et Perdue 1992). A la suite de Turco (2008) et de Sun (2008) nous avons sélectionné la séquence du «vol du pain », qui permet d'observer l'encodage de plusieurs personnages qui apparaissent et reviennent sur la scène. Pour cette étude, nous avons ultérieurement manipulé la séquence en y ajoutant une scène dans laquelle le personnage de la «voleuse » se rend auprès du commissariat où se trouve Chaplin pour le 
retrouver en lui faisant une surprise. De cette manière nous avons pu étudier la façon dont un référent est réintroduit dans le discours suite à une interruption narrative.

Nous avons sélectionné quinze participants sinophones, locuteurs de chinois mandarin, qui ont un niveau de français avancé 3 . Ce groupe de locuteurs a produit une narration en chinois L1 et une en français L2. Afin de permettre une comparaison avec les productions en langue cible, nous avons recueilli aussi des narrations parallèles produites par quinze locuteurs francophones natifs, qui nous ont servi comme groupe de contrôle. Au total, nous disposons ainsi de trois corpora : français L1, chinois L1 et français L2.

Nos informateurs ont répondu à un questionnaire sociolinguistique permettant de recueillir des informations concernant la quantité et le type d'exposition à la L2. Les apprenants sélectionnés ont appris la L2 en milieu universitaire et ont une connaissance métalinguistique profonde de la langue cible. La période d'immersion linguistique est de plus de 3,5 ans en moyenne. La plupart des participants sinophones vivent en France depuis plusieurs années, où ils pratiquent la L2 au quotidien, notamment dans le contexte académique. Etant donné la taille très réduite de notre corpus, l'analyse des données est essentiellement qualitative, et les chiffrages ne permettent qu'une estimation indicative des tendances.

\section{Description des outils syntaxiques associés à l'introduction référentielle en français et chinois $L 1$}

Nous nous intéressons aux structures syntaxiques particulières qui interviennent pour introduire un référent dans l'univers du discours narratif. Le principal dispositif syntaxique dédié à cette tâche est la structure présentative en AVOIR, tant en français (Léard 1986, Lambrecht 1987b, Ashby 1995 inter alia), qu'en chinois (Chao 1968: 727-729, Li et Thompson $1981: 509$, Huang $2013: 244$ ).

Par ailleurs, on relève d'autres structures ayant la même fonction d'introduction d'un référent. Sur la base des configurations pertinentes à l'analyse de nos données, nous présentons ici notamment les phrases existentielles « simples » (sans subordonnée relative : «il y $a+\mathrm{SN}$ ») et les structures présentatives (comportant une subordonnée relative : "il y $a$ $+\mathrm{SN}+q u i \ldots »)$ centrées sur le verbe AVOIR, les présentatives de PERCEPTION (du type « on voit SN qui... ») ainsi que l'inversion du verbe principal - produisant l'ordre verbe-sujet (ex. : « devant elle s'approche quelqu'un »). Le chinois et le français partagent toutes ces structures, mais avec pourtant parfois certaines spécificités d'emploi, que nous présentons dans la section suivante.

\subsection{Structures existentielles et présentatives en AVOIR dans les deux L1}

Le chinois et le français font partie des langues qui s'appuient sur le verbe AVOIR pour marquer l'existence, comme l'espagnol, le grec, le bulgare (Heine et Kuteva 2005 : 33). De plus, comme c'est souvent le cas, ces deux langues ont développé une construction où la formule existentielle est utilisée au sein d'une structure bipartite pour introduire un syntagme

\footnotetext{
${ }^{3}$ Les locuteurs sinophones interrogés étudient le français depuis plus de 6 ans en moyenne. Sur 15 participants, seuls 3 ne vivaient plus en France au moment de l'enregistrement, mais y ont résidé plus d'un an et ils continuent de pratiquer la langue cible - pour cette raison nous n'avons pas estimé nécessaire de départager les productions des locuteurs se trouvant en condition d'immersion linguistique au moment de l'enregistrement de ceux qui ne l'étaient plus. Il aurait été sûrement préférable de faire appel à deux groupes de locuteurs sinophones, les uns produisant une narration dans leur langue maternelle et les autres dans la langue cible, cependant, pour des raisons d'ordre pratique cela n'a pas été possible ; nous avons décidé de recourir à un groupe de locuteurs unifié qui a produit une narration et en L1 et en L2. Je remercie un relecteur anonyme pour sa remarque à ce propos.
} 
nominal dénotant une entité nouvelle (Creissels, à paraître). Ainsi en français, de façon générale, la fonction d'introduction référentielle est confiée aux structures présentatives en AVOIR, notamment aux tournures en il y a SN qui. Lorsqu'il n'y a pas de relative suivant le syntagme nominal, on parlera de phrases existentielles « simples » (sauf indication contraire, les exemples proviennent de notre corpus) :

(4) FR1 ils sont tous les deux par terre
il y a un attroupement et ya un policier qui arrive
[Structure « existentielle »] [Structure «présentative »]

La séquence existentielle centrée sur le verbe AVOIR peut prendre la forme impersonnelle (il) y a ou bien se construire avec un pronom de personne «j'ai/t'as...qui... » (Conti 2010), comme dans l'énoncé suivant (nous n'avons que ce seul exemple de « j'ai/t'as » $+\mathrm{SN}+q u i \ldots$ dans notre corpus des francophones natifs et aucune occurrence n'est relevée chez les apprenants sinophones de français L2) :

(5) FR1

\author{
t'as le boulanger \\ ou en tout cas quelqu'un qu'y travaille \\ qui vient déposer le pain frais \\ vient déposer le pain frais
}

En chinois, langue n'ayant pas de pronoms relatifs ${ }^{4}$, ce qui distingue la formule simple (6) de la complexe (7) est ainsi la présence d'un deuxième verbe dont le SN introduit par yǒ 有 'avoir' dénote l'agent sémantique :

(6) $\mathrm{CH} 1$
旁边有很多围观群众
[Structure « existentielle »]
Pángbiān yǒu hěnduō wéiguān qúnzhòng
À.côté AVOIR beaucoup spectateur gens
'À côté il y a un groupe de curieux'

(7) $\mathrm{CH1}$

\title{
看! 有人偷你的面包！～[Structure 《 présentative »] \\ Kàn! Yŏu rén tōu nīde miànbāo! \\ Regarder AVOIR personne voler ton pain \\ 'Regarde ! Il y a quelqu'un [qui] a volé ton pain !'
}

Parfois, un pronom de personne vient s'insérer entre le syntagme nominal et le verbe : nous analysons le type d'énoncé en (8) comme une « construction présentative ». Au niveau prosodique, il s'agit d'une structure unitaire puisqu'il n'y a pas de pause entre le SN et le pronom.

(8) $\mathrm{CH} 1$

\section{有一个人他送面包进来 \\ Yŏu yí-ge rén tā sòng miànbāo jìn-lai}

\footnotetext{
${ }^{4}$ La particule $d e$ 的, se situant à gauche du nom qu'elle modifie, peut introduire des propositions « imbriquées » :

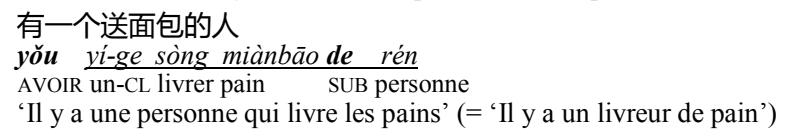

Si cette forme est possible, la particularité des «structures en yǒu » est justement celle de présenter d'abord le $\mathrm{SN}$ et ensuite l'information à son propos. Dans l'exemple (I) de 的 est traduit par le relatif qui mais pour être précis cette particule « est un morphème vide, un opérateur de nominalisation, auquel on ne peut attacher aucune étiquette sémantique » (Paris 1977). Dans notre corpus de chinois L1, les structures « présentatives » en yǒu comportent toutes la séquence yǒ $+\mathrm{SN}+\mathrm{SV}$.
} 
AVOIR un-CL personne 3s livrer pain entrer-venir

Lit: 'Il y a quelqu'un il est rentré amener du pain'

Cette insertion pronominale témoigne à nos yeux d'un marquage explicite de la structuration de l'information en deux blocs : au premier segment introduit par yǒ ' 'avoir' est confiée la fonction de présenter l'entité dénotée par le $\mathrm{SN}$, tandis que la deuxième séquence guidée par le pronom personnel, qui reprend anaphoriquement le référent du SN, porte sur la prédication à son propos. Ceci est particulièrement notable au sein d'une langue telle que le chinois où l'expression des pronoms personnels en position sujet relève d'un choix optionnel. Les structures «présentatives » se font au moyen d'un clivage syntaxique pour répondre à la contrainte cognitive qui empêche le cumul de la fonction présentative et de la fonction prédicative au sein d'une même structure argumentale (obéissant ainsi au Principle of the Separation of Reference and Role élaboré par Lambrecht 1994 : 184); l'insertion pronominale ne fait que souligner une telle articulation de la proposition en deux noyaux prédicatifs.

Si une analyse de ce type de phrase n'a pas été faite pour le chinois, beaucoup d'auteurs se sont intéressés à la forme il y a SN $i l(s) \ldots$ du français L1 (Jeanjean 1979 : 132 ; Berrendonner 2003 : 260 ; Choi-Jonin et Lagae 2005 inter alia), dans laquelle donc le pronom personnel prend la place du relatif qui :

\section{(9) FR1 Allez ah Nadia vite ah speed vite il y a le bus il va partir ${ }^{5}$}

Cappeau et Deulofeu (2001) estiment que cette forme relève d'une structuration opérant au niveau discursif en termes de topique-commentaire plutôt que se situer au niveau grammatical en tant que relation sujet-prédicat, telle que propre à la forme il y a...qui. L'apparition d'une structure de ce type chez les apprenants sinophones de français pourrait répondre à ces mêmes principes d'organisation discursive, en général plus proche du système « topique-commentaire » du chinois L1 (section 4.2).

En somme, les structures présentatives en AVOIR des deux L1 se différencient sur le plan morphosyntaxique dans la mesure où le chinois, n'ayant pas de conjugaison verbale, ne demande pas d'accord entre le SN et le syntagme verbal qui le suit; le verbe yǒu 'avoir' par conséquent est invariable, contrairement à la formule du français (« il y a/avait»; "j'ai, t'as/avais » etc.) ; la structure du chinois ne comporte pas non plus de pronom relatif.

Du point de vue constructionnel, on remarquera que le français admet des SN définis au sein de ses formules présentatives en avoir, incluant les noms propres (10) et les noms communs avec un possessif ou un démonstratif (Verwimp et Lahousse 2016).

En revanche, une forte contrainte de définitude pèse sur les structures en yǒu du chinois (Cai 2000 ; Hu et Pan 2008), car le chinois n'admet pas de SN définis au sein de ses structures présentatives en AVOIR. Confrontons les deux énoncés suivants, en français et en chinois L1 :

(10)FR1

(11) $\mathrm{CH} 1$
Y a Jean qui est venu

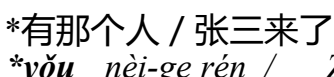

*yǒu nèi-ge rén / Zhāngsān lái-le

AVOIR ce-CL personne Zhangsan venir-PFV

Sens visé : 'Il y a cet homme / Il y a Zhangsan qui est venu'
(Côté 1998)

(Hu et Pan 2008)

Du point de vue pragmatique, conformément à leur fonction principale d'introduction d'un référent dans le discours, les constructions présentatives sont utilisées en priorité avec des référents ni identifiables ni ancrés. Précisons que la construction clivée en avoir exprime

\footnotetext{
${ }^{5}$ Exemple tiré de Williems et Meullemen (2010) reporté par Karssenberg et Lahousse (2016). Certes, la structure il y a $\mathrm{SN}$ il(s)... est connotée stylistiquement et ne serait pas acceptée en français standard (je remercie un relecteur anonyme pour cette remarque).
} 
le plus souvent ${ }^{6}$ une articulation informative de type «all-focus » (dénotant une proposition « thétique »), laquelle ne comporte ni topique ni arrière-plan discursif.

A cet égard, il est utile de faire une distinction entre les dimensions référentielle et relationnelle de la structure informative, la première étant liée à l'accessibilité des référents tandis que la deuxième tient au rapport entre les éléments au sein d'une proposition. Une expression peut notamment dénoter un référent déjà évoqué, tout en exprimant en même temps de l'information nouvelle (Bentley et al. 2015:45; Karssenberg à paraître). C'est pourquoi, tout en montrant une prévalence des référents non identifiables, les structures présentatives en il y a peuvent aussi bien enchâsser des référents identifiables ou déjà introduits (Ashby 1995 ; Lambrecht 1988 : 167-69).

Or en chinois, les structures en yǒu ne peuvent que très difficilement comporter des SN renvoyant à des référents identifiables - comme le montre l'agrammaticalité de (11). Ainsi, les structures présentatives en AVOIR dans cette langue sont soumises à des contraintes pragmatiques plus strictes: non seulement donc elles apparaissent sous un contexte énonciatif de nature thétique mais il est tout aussi nécessaire que le SN focalisé dénote un référent entièrement inaccessible - nouveau à la fois sur le plan référentiel et relationnel. Lorsqu'un référent est accessible, que ce soit en raison du contexte linguistique (tel que le cas des référents réintroduits) ou extralinguistique (SN à référence unique), a priori on n'aura pas d'encodage via la structure présentative en AVOIR ${ }^{7}$.

En tant que natifs, les locuteurs chinois ont intériorisé ces facteurs conditionnant l'usage des structures présentatives dans leur langue source. On peut se demander comment les apprenants sinophones se comporteront face aux subtiles différences que nous venons d'exposer quant aux principes pragmatiques qui guident l'usage des structures en AVOIR dans la langue cible.

\subsection{D'autres configurations utilisées pour l'encodage référentiel en L1}

\subsubsection{Introduction référentielle faite au moyen d'un verbe de PERCEPTION en L1}

Nous nous référons ici aux structures ayant la forme de base « on voit SN (qui SV) ». Il s'agit des constructions relatives présentatives de PERCEPTION (Lambrecht 2000), où le prédicat principal est caractérisé par la présence d'un élément de perception. En français, le SN est en même temps le sujet de la relative :

\section{(12) FR1 On peut voir une jeune fille qui se promène dans la rue}

Le chinois présente également ce type de structure où le SN dénotant l'entité introduite apparaît en tant qu'objet d'un verbe de perception, mais en l'absence de pronom relatif en chinois, cette structure peut donner lieu à une suite de prédicats (exemple 13a), ou à une

\footnotetext{
${ }^{6}$ L'apport de la linguistique de corpus nous montre en effet que les clivées en il y a expriment le plus souvent une articulation de type «all-focus ». D'autres types d'articulations focales sont recensées, à savoir la structure focusbackground où l'élément focalisé est le SN introduit par il y a tandis que la relative rentre dans le domaine de la présupposition - ressortant premièrement dans des contextes de question/réponse - et celle "à double contraste », plus rare (Karssenberg à paraître). Dans notre corpus nous relevons seul le type le plus courant « all-focus », ayant notamment la fonction de " présenter » une entité référentielle.

${ }^{7}$ Bien sûr, il y a des exceptions, notamment lorsqu'un adverbe focalisateur tel que hái 还 'aussi' précède le verbe yǒu 有; ici la structure aurait la fonction de marquer une nouvelle relation au lieu d'introduire une entité dans le discours (Hu et Pan 2008). Par ailleurs, nous n'avons pas dans ce cas de deuxième prédicat donc il s'agit toujours de structures existentielles « simples ». D’autres auteurs ont remarqué que la structure en yǒu peut parfois enchâsser des $\mathrm{SN}$ définis, il s'agit toutefois de cas rares et apparaissant dans des contextes soumis à des nombreuses contraintes (Cai 2000).
} 
tournure où le référent dénoté par le SN est repris au moyen d'un pronom personnel (13b), de manière similaire aux structures présentatives en AVOIR.

(13) a. CH1 然后见到一个面包师在搬运面包

Ránhòu jiàndào yí-ge miànbāoshā zài bānyùn miànbāo

Après voir un-CL boulanger PROG déplacer pain

'Après [elle] voit un boulanger [qui] est en train de déplacer les pains'

b. CH1 我看到一个女儿她看上是很饿

Wó kàndào yí-ge nǘér tā kànshàng shì hèn è

$1 \mathrm{~S}$ voir un-CL fille $3 \mathrm{~S}$ apparaître être très affamé

'Je vois une fille elle a l'air d'être affamée'

Les présentatives de PERCEPTION sont d'ailleurs dans nos données moins fréquentes chez les sinophones que dans les productions des francophones. Dans notre étude, nous incluons les cas où l'on n'a pas de proposition relative, puisqu'il s'agit d'une tournure utilisée par les apprenants sinophones de français L2 (section 4.3.2).

\subsubsection{Structures présentatives du type ETRE + SN dans les L1}

Au-delà des structures en il $y a$ et des présentatives de PERCEPTION, les locuteurs francophones natifs peuvent également s'appuyer sur l' « introducteur » (Leclercq 2008) c'est pour ancrer une nouvelle entité référentielle dans le discours. Nous nous référons notamment aux structures en c'est ... (qui) "à focus large », dans lesquelles la phrase entière est rhématique (14a). Elles se distinguent des formes en c'est «à focus étroit » où seulement le $\mathrm{SN}$ est focalisé, tandis que la relative rentre dans le domaine de la présupposition (14b).

(14) a. FR1 Alors c'est une jeune fille qui est pauvre

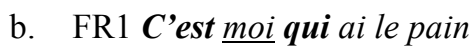

Parmi les structures en $c$ 'est à focus large nous incluons la clivée «à enchaînement » (Scappini 2014), où l'information nouvelle n'est pas celle qui suit c'est mais celle encodée par la relative (exemples en 15). Dans l'énoncé (15b) la structure en c'est «à focus large » est utilisée non pour introduire un nouveau référent mais pour présenter un événement sous un contexte thétique, qui à son tour inclut une structure en c'est « à fonction identificatrice ».

(15) a. FR1 c'est alors que le boulanger arrive et qu'un policier arrive

b. FR1 c'est alors que Charlie Chaplin prétend que c'est lui qui a volé la baguette

En chinois L1 l'introduction des nouveaux référents peut également se réaliser au moyen de la copule shi 是 'être', et ces constructions comportent souvent une suite de deux prédicats (dans l'exemple qui suit avec l'insertion d'un pronom personnel) :

(16) $\mathrm{CH} 1$ 首先呢是一个年轻的女孩子她看到橱窗里面 Shǒuxiān ne shì yí-ge niánqīng de nüháizi tā kàndào chúchuāng lǐmiàn D'abord NE être un-CL jeune SUB fille 3S voir vitrine dedans 'Au début [c']est une jeune fille elle regarde dans la vitrine'

Tout comme pour les présentatives de PERCEPTION, les deux L1 se distinguent premièrement par leur recours très inégal à cette structure, puisqu'en chinois il s'agit d'un 
procédé marginal tandis qu'elle est couramment utilisée en français L1. En général, dans nos données les francophones natifs font appel à un inventaire plus diversifié de structures présentatives, alors que dans les narrations des sinophones la fonction d'introduction référentielle faite au moyen d'un introducteur est premièrement confiée aux structures en AVOIR. D'autre part, les locuteurs chinois se servent fréquemment de l'inversion de l'ordre des mots, procédé possible chez les francophones également, avec pourtant quelques différences que nous présentons dans la section suivante.

\subsubsection{Ordre verbe-sujet en français et chinois $L 1$}

Contrairement à d'autres langues romanes telles que l'italien et l'espagnol, en français la postposition du sujet nominal au verbe est assez contrainte et relève principalement du registre écrit ; à l'oral, on rencontre parfois des sujets postposés mais dans des contextes assez précis. La principale fonction de l'ordre V-S en français est de focaliser le référent dénoté par le sujet postposé et certains verbes, notamment des verbes de déplacement tels que arriver, entrer, sortir, passer apparaissent fréquemment avec l'inversion nominale (Cappeau et Lahousse 2015). Nous avons rencontré quelques exemples d'ordre V-S dans notre petit corpus de français $\mathrm{L}^{8}{ }^{8}$; il s'agit majoritairement de cas d'inversion locative (comportant un complément spatio-temporel antéposé) :

\section{(17) FR1 Devant elle s'approche quelqu'un}

En chinois, la structure en V-S est une formulation syntaxique assez répandue, à l'écrit comme à l'oral, ayant notamment la fonction de présenter une nouvelle entité référentielle dans la scène du discours ( $\mathrm{Li}$ et Thompson 1981 : 517 ; Huang 2013 : 244). Dans cette langue, on peut avoir aussi une inversion locative, qui comporte un SN dénotant la référence spatiotemporelle en tête de phrase ou une inversion " absolue », spécifique notamment à un sousgroupe de verbes que l'on peut qualifier d' « inaccusatifs » (verbes de déplacement, verbes dénotant un changement d'état etc.). Ainsi, comme en français, les nouveaux référents sont souvent introduits par des verbes de déplacement présentatifs tels que les déictiques lái 来, guòlai 过来 ‘venir' et ceux dénotant «l'apparition » comme chüxiàn 出现 'apparaître' :

CH1 这个时候来了一位警察
Zhège shíhou lái-le yí-wei jĭngchá
ce-CL moment venir-PFV un-CL policier
就问发生什么事情
jiù wèn fāshēng shénme shiquing
donc demander arriver quel affaire
'A ce moment-là arrive un policier demandant qu'est ce qui s'était passé'

Tout comme pour les structures en yŏu 'avoir', une forte restriction de définitude pèse sur les énoncés en V-S du chinois. Notamment, les SN postverbaux sont principalement des noms nus ou des noms accompagnés par la séquence [numéral + classificateur], deux

\footnotetext{
${ }^{8}$ Ces résultats concernant l'ordre V-S en français L1 semblent aller à l'encontre de ceux obtenus par Turco dans son étude de 2008 : « chez les sujets francophones, nous ne retrouvons pas de cas d'inversion V-S en présence de V inaccusatifs : l'introduction d'un référent dans la scène dans un contexte énonciatif thétique est réalisée toujours pas l'ordre S-V (+ emploi de connecteurs) ». Cette divergence de résultats tient vraisemblablement à la taille réduite des corpora en regard de la grande variabilité quant aux choix des tournures syntaxiques associées à l'introduction référentielle est sans doute à la base de cette divergence. Pour cette même raison, les chiffres présentées dans notre travail n'aspirent nullement à démontrer la représentativité de nos données, mais nous fournissent juste un cadre indicatif pour conduire notre analyse.
} 
formulations qui équivalent grosso modo à un syntagme nominal indéfini en français. En français, en revanche, l'ordre V-S peut tout à fait s'appliquer à des SN postverbaux définis (dans l'exemple qui suit un nom propre) :

\section{(19) FR1 De l'autre côté de la rue vient Charlot qui marche}

Il est notable que dans cet exemple d'ordre V-S en français L1, le SN est repris par une subordonnée relative. Sur l'importance de la reprise relative dans la structuration du discours chez les francophones, en rapport aux productions des sinophones, on peut se référer à la section 4.5.

En conclusion, nous constatons que, bien que le français et le chinois L1 soient deux langues typologiquement distantes, elles partagent tout un inventaire de constructions « présentatives » qui ont pour fonction d'introduire des nouveaux référents dans le discours. En particulier, les tournures clivées en AVOIR sont un procédé largement utilisé chez les francophones et les sinophones, à l'écrit comme à l'oral, qui permet de rendre saillant le référent dénoté par le SN. Ce qui distingue les structures de ces deux langues sur le plan morphosyntaxique, c'est tout d'abord l'absence de pronom relatif en chinois. Au niveau fonctionnel, nous avons montré que les constructions présentatives en AVOIR du chinois ne peuvent pas enchâsser de référents " déjà activés », comme le font les structures du français, et que cette différence se manifeste dans la restriction de définitude affectant l'apparition des $\mathrm{SN}$ au sein de la structure en yǒu.

D'autre part, les structures présentatives comportant un verbe de PERCEPTION sont peu utilisées par les sinophones, alors que l'ordre V-S est employé chez les locuteurs natifs des deux langues pour introduire les nouveaux référents dans le discours. Cependant, tandis qu'en français cette configuration relève en général d'un registre soutenu, il est fréquemment utilisé en chinois, sans contrainte de niveau de langue. En revanche, l'ordre V-S ne peut pas en chinois comporter des SN définis, le français L1 n'ayant pas cette limitation.

En somme, les structures «présentatives» en AVOIR des deux L1 présentent une remarquable corrélation forme/fonction, avec néanmoins des différences sur les plans syntaxique (absence de pronom relatif en chinois L1), constructionnel (restriction de définitude) et pragmatique (par rapport au statut référentiel du SN focalisé). La maîtrise de telles structures dans la langue cible demande aux apprenants de s'adapter à toutes ces contraintes relevant de différents niveaux linguistiques.

En général, nous nous attendons à ce que l'emploi des tournures présentatives en AVOIR chez les apprenants sinophones soit facilité par la présence du même type de structure dans leur L1. En même temps, la nature polyfonctionnelle des clivées en il y a de la langue cible peut vraisemblablement poser des problèmes, dans la mesure où les apprenants doivent reconsidérer les corrélations forme/fonction(s) pour acquérir un usage de ces constructions conforme à celui des natifs. Pour ce qui est de l'ordre V-S à fonction présentative, les apprenants sinophones devraient l'utiliser aisément parce qu'ils disposent de ce procédé dans leur langue source. D'autre part, le fait qu'un tel arrangement des mots est moins fréquent en français oral pourrait également avoir de l'influence sur les choix des apprenants.

Les résultats sont discutés dans la section suivante.

\section{Résultats}

\subsection{Les diverses structures syntaxiques associées à la fonction d'introduction référentielle}




\subsubsection{Structures " présentatives » et ordre sujet-verbe en L1 et L2}

Dans cette partie, nous présentons brièvement l'analyse quantitative des données relatives à chaque groupe de locuteurs pour ensuite passer à une analyse qualitative de ces emplois de la part des apprenants sinophones. Les Tableaux 1-3 donnent une vue d'ensemble de la distribution des structures « présentatives » (toute structure syntaxique associée à la fonction d'introduction référentielle) dans les textes des natifs francophones et sinophones ainsi que dans les récits en français L2. Les pourcentages sont calculés sur le total des propositions dans le Tableau 1 et sur le total des structures syntaxiques encodant la première expression d'un référent dans les Tableaux 2 et 3 (à l'exclusion des énoncés où le nouveau référent apparaît en tant qu'objet du verbe ${ }^{9}$ ).

Tableau 1. Occurrence absolue et relative (\% sur le total des propositions) des structures présentatives dans les productions des natifs et des apprenants sinophones

\begin{tabular}{|l|c|c|c|}
\hline & Français L1 & Chinois L1 & Français L2 \\
\hline Total des propositions & 589 & 591 & 652 \\
\hline $\begin{array}{l}\text { Structures présentatives } \\
\text { (\% nombre total de propositions) }\end{array}$ & $47 \mathbf{( 7 . 9 \% )}$ & $50(\mathbf{8 . 4 \% )}$ & $35(\mathbf{5 . 3 \% )}$ \\
\hline
\end{tabular}

En général, sur le nombre total des propositions, les sinophones et les francophones natifs produisent environ $8 \%$ de structures présentatives, tandis que les apprenants sinophones de français L2 en produisent un peu moins (5\%).

Le tableau suivant présente la distribution des structures présentatives et à ordre non marqué sujet-verbe, employées chez les natifs et les apprenants pour introduire un nouveau référent dans la narration :

Tableau 2. Distribution des structures présentatives et à ordre non marqué $\mathrm{S}-\mathrm{V}$ (\% sur le total des propositions incluant un nouveau référent)

\begin{tabular}{|l|c|c|c|}
\hline & Français L1 & Chinois L1 & Français L2 \\
\hline Structures présentatives & $47(\mathbf{7 1 \%})$ & $50(\mathbf{6 6 \%})$ & $35(\mathbf{5 2 \%})$ \\
\hline Ordre non marqué sujet-verbe & $19(\mathbf{2 9 \%})$ & $26(\mathbf{3 4 \%})$ & $32(\mathbf{4 8 \%})$ \\
\hline TOTAL & $66(100 \%)$ & $76(100 \%)$ & $67(100 \%)$ \\
\hline
\end{tabular}

Lorsque les apprenants sinophones de français doivent introduire une nouvelle entité référentielle, ils ont recours de façon importante aux tournures à ordre $\mathrm{S}-\mathrm{V}(48 \%$ en français L2 contrairement à $29 \%$ et $34 \%$ des occurrences en français et chinois L1, respectivement).

Le Tableau 3 recense plus en détail la répartition des structures syntaxiques associées à l'expression des nouvelles entités référentielles dans les narrations en français et chinois L1 ainsi qu'en français L2.

Tableau 3. Distribution des structures syntaxiques associées à la fonction d'introduction référentielle (\% sur le total des propositions incluant un nouveau référent)

\begin{tabular}{|l|l|l|l|}
\hline & Français L1 & Chinois L1 & Français L2 \\
\hline AVOIR présentatif & $\mathbf{2 0 ( 3 0 \% )}$ & $\mathbf{2 6 ( 3 4 \% )}$ & $11(16 \%)$ \\
\hline
\end{tabular}

\footnotetext{
${ }^{9}$ La position de complément d'objet postverbal étant l'emplacement focal non marqué tant en français qu'en chinois, nous n'incluons pas ces énoncés dans notre traitement, étant donné qu'ils ne s'opposent pas à des encodages syntaxiques alternatifs.
} 


\begin{tabular}{|l|l|l|l|}
\hline AVOIR existentiel & $2(3 \%)$ & $10(13 \%)$ & $13(19 \%)$ \\
\hline Présentative de PERCEPTION & $11(17 \%)$ & $4(5 \%)$ & $9(14 \%)$ \\
\hline$V-S$ & $6(9 \%)$ & $8(11 \%)$ & $0(0 \%)$ \\
\hline ETRE $+S N$ & $8(12 \%)$ & $2(3 \%)$ & $2(3 \%)$ \\
\hline$S_{\text {Défini- } V}$ & $13(20 \%)$ & $13(17 \%)$ & $12(18 \%)$ \\
\hline$S_{\text {Indéfini- } V}$ & $6(9 \%)$ & $13(17 \%)$ & $\mathbf{2 0 ( 3 0 \% )}$ \\
\hline TOTAL & $66(100 \%)$ & $76(100 \%)$ & $67(100 \%)$ \\
\hline
\end{tabular}

Nous avons relevé les structures suivantes : les tournures en AVOIR « existentielles» («il $\mathrm{y}$ a un policier ») et " présentatives »- suivies par un deuxième prédicat ( « il y a un policier qui arrive »); l'ordre « non marqué » sujet-verbe classé selon que le SN préverbal est défini ( "la police arrive ») ou indéfini ( « un policier arrive ») ; la présentative de PERCEPTION ( voit un policier (qui arrive) ») ; l'ordre « inversé » verbe-sujet (« arrive un policier ») et les phrases construites autour du verbe ETRE («c'est un policier (qui arrive) ») où le SN apparaît notamment au sein de la structure en c'est... (qui) « à focus large » en français L1 et L2 et en tant qu'argument du verbe copulatif en chinois L1. Nous n'avons pas inclus les cas où un référent est introduit dans le discours en tant qu'objet du verbe (ex : « il a appelé la police »).

Tout d'abord, les résultats montrent que chez les sinophones et les francophones natifs, les structures présentatives en AVOIR représentent effectivement la tournure la plus fréquemment utilisée pour encoder un nouveau référent (exemple 20 et 21 respectivement) :

$$
\begin{aligned}
& \mathrm{CH} 1 \text { 有一个先生从车子里拿出了一些东西 } \\
& \text { Yóu yí-ge xiānsheng cóng chēzi=li ná-chu-le yìxie dōngxi } \\
& \text { AVOIR un-CL monsieur depuis voiture=dans prendre-sortir-PFV quelques choses } \\
& \text { 'Il y a un monsieur [qui] a sorti des choses du camion' }
\end{aligned}
$$

\section{FR1 à ce moment-là il y a le camion qui vient livrer les gâteaux}

De plus, on constate chez les francophones un usage très réduit des structures existentielles «simples» (autrement dit, sans relative co-indexée avec le SN - seules 2 occurrences totales), contrairement aux sinophones, chez lesquels le verbe yǒu 'avoir' est plus fréquemment utilisé conjointement avec un deuxième prédicat, mais se trouve également en tant que prédicat unique (26 et 10 occurrences, respectivement).

Dans le corpus des apprenants, en revanche, nous observons que la priorité est donnée aux phrases suivant l'ordre non-marqué $\mathrm{S}-\mathrm{V}$ (exemple 22a), et seulement dans un deuxième temps aux phrases existentielles (22b) et aux structures présentatives (22c) en AVOIR.

\section{(22) a. FR2 Une jeune fille marche dans la rue elle avait très faim \\ b. FR2 Il y a une fille sans domicile \\ c. FR2 Donc, il y avait une femme qui est passée devant une boulangerie}

On remarquera notamment l'emploi de l'ordre S-V avec un sujet préverbal indéfini (ex. 22a), nettement plus important chez les apprenants qu'en français et en chinois L1.

En général, les apprenant ont recours à un ensemble moins diversifié de structures présentatives par rapport aux francophones natifs. Nous relevons seulement deux cas de structures en c'est à focus large et 9 occurrences de présentatives faites au moyen d'un verbe de PERCEPTION (section 4.3.2). Aucun exemple d'ordre V-S n'est relevé chez les apprenants 
sinophones, tandis qu'il est attesté dans les corpora des locuteurs natifs (6 occurrences en français L1 et 8 en chinois L1). Nous discuterons en détail de cet aspect dans la section 4.3.1.

En somme, un premier constat que nous pouvons faire est que la fréquence d'usage des structures présentatives en AVOIR est moins importante chez les apprenants : la présence des structures en yǒ dans leur langue source ne semble pas favoriser l'usage des tournures en AVOIR dans les productions en français L2.

\subsubsection{Structures syntaxiques employées pour introduire le référent de la dame " moucharde " chez les natifs et les apprenants}

Dans ce qui suit, nous analysons plus en détails la manière dont est codée la première expression de l'un des référents, notamment celui de la dame «moucharde », car son encodage diffère sensiblement dans les deux L1 et dans les productions des apprenants.

Dans cette scène, la protagoniste prend la fuite après avoir volé une baguette dans une boulangerie. Cependant, une dame la voit et se précipite pour prévenir le boulanger. C'est à cause de cette dame « moucharde » que la protagoniste principale sera accusée de vol.

A nos yeux, l'expression particulière de ce référent dans les productions des locuteurs natifs est motivée par le contexte narratif. En effet, le personnage de la dame « moucharde» apparaît sur la scène du discours de façon inattendue - sous un contexte purement thétique et en raison de son action, ce qui bloquerait plus nettement un encodage de type $\mathrm{S}-\mathrm{V}$ en français L1. Dans les productions des sinophones natifs, en revanche, le rôle sémantique prend le dessus sur la structuration discursive, notamment car l'utilisation du passif en chinois, qui sous-entend généralement une expérience désagréable, est en parfaite adéquation avec la situation décrite dans ce passage de la vidéo. Les résultats sont présentés dans le Tableau 4 (le nombre total d'occurrences pour chaque groupe varie en raison d'une reformulation - en français L2, et du fait de l'absence de ce référent dans deux narrations chez les francophones natifs) :

Tableau 4. Distribution des structures syntaxiques employées pour introduire le référent de la dame « moucharde » chez les locuteurs natifs et les apprenants sinophones

\begin{tabular}{|l|c|c|c|}
\hline & Français L1 & Chinois L1 & Français L2 \\
\hline Structure en AVOIR & $\mathbf{1 0}$ & 2 & 3 \\
\hline Ordre $S-V$ & 3 & 4 & $\mathbf{9}$ \\
\hline Ordre $V-S$ & -- & 1 & -- \\
\hline Construction passive & -- & $\mathbf{8}$ & 4 \\
\hline OCCURRENCES TOTALES & 13 & 15 & 16 \\
\hline
\end{tabular}

Comme le montre le tableau 4, chez les locuteurs francophones l'introduction de ce référent est réalisée systématiquement avec la structure présentative en AVOIR (exemple 23), exception faite de trois cas d'ordre S-V. Les sinophones, en revanche, recourent le plus souvent à la construction passive, où le $\mathrm{SN}$ dénotant le référent visé est introduit par la particule bèi 被 (exemple 24):

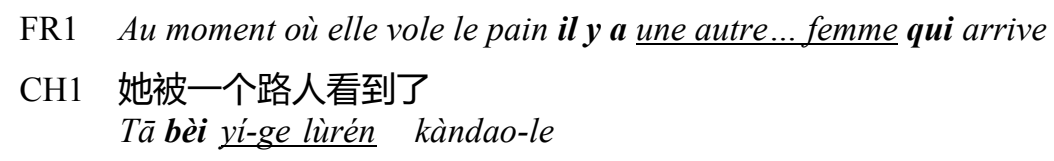

FR1 Au moment où elle vole le pain il y a une autre... femme qui arrive

$\mathrm{CH} 1$ 她被一个路人看到了 Tà bèi yí-ge lùrén kàndao-le 
3S PASS Un-CL passant voir-PFV

'Elle a été vue par une passante'

Dans ce cas, le choix des apprenants diverge manifestement de ceux des francophones natifs, d'un côté, et des productions en chinois L1 de l'autre. Si on relève quelques occurrences de structure en avoir (exemple 25a) et de construction passive, laquelle par ailleurs est absente dans le corpus de francophones natifs (exemple 25b), les locuteurs sinophones rétablissent le plus souvent l'ordre S-V en français L2 (ex. 25c) :

(25) a. FR2 derrière elle il y avait une femme plus âgée est arrivée

b. FR2 et elle est surprené par une autre femme

c. FR2 une dame qui passe l'a vue

Ces données nous montrent de façon plus nette comment le choix des apprenants tend vers un troisième pôle, qui n'est ni la construction passive ni la structure présentative en avoir, lesquelles sont nettement préférées en chinois et français L1, respectivement. Ainsi, le fait d'employer l'ordre sujet-verbe en français L2 dans ce contexte témoigne sans doute à la fois d'un manque de maîtrise pragmatique et d'une conceptualisation de cet ordre des mots en tant que séquence « canonique » de la langue cible.

Dans ce qui suit, nous allons analyser en détail les diverses structures «présentatives » associées aux productions des apprenants, à savoir les structures en AVOIR (section 4.2), l'ordre V-S (4.3.1), les présentatives de PERCEPTION (4.3.2) et les structures en $c$ 'est « à focus large » (4.3.3). Par la suite nous nous intéresserons aux tournures syntaxiques liées à la fonction de réintroduction référentielle, et verrons notamment que chez les apprenants une telle fonction ne peut pas s'exprimer au travers des structures présentatives, contrairement aux francophones natifs (4.4). En dernier, nous prendrons en considération l'ensemble des structures ayant la fonction de (ré)introduction référentielle en rapport au contexte à la droite du SN qu'elles enchâssent, ce qui nous permettra de confronter les divers procédés adoptés en français L1 et L2 pour assurer la reprise du SN (ré)introduit (4.5).

\subsection{Caractéristiques des structures présentatives en AVOIR chez les apprenants sinophones}

Maintenant, nous observons de plus près les caractéristiques des structures en AVOIR chez les apprenants sinophones. Premièrement, nous avons constaté une préférence pour les phrases existentielles « simples », fait qui contraste avec les productions en français L1 (seules deux occurrences chez les francophones natifs).

Dans une étude de 2002, Chini analyse la production en italien L2 d'une jeune locutrice sinophone, laquelle « recourt à une proportion importante de formes « simples » esserci + Sujet sans continuation pseudorelative $(69 \%)$, et à une proportion réduite de formes présentatives avec continuation pseudorelative $(21 \%) »$. La tendance à préférer les structures simples sans reprise relative semble ainsi se rencontrer aussi chez les apprenants sinophones de niveau peu avancé.

En outre, nous relevons dans le corpus des apprenants des structures présentatives en AVOIR qui comportent l'omission du pronom relatif qui. Dans les exemples suivants en français L2, le SN encodé en tant qu'objet au sein de la structure présentative en AVOIR est suivi directement par le deuxième prédicat qu'il régit :

\section{(26) a. FR2 derrière elle il y avait une autre dame ol'a vue déjà \\ b. FR2 pendant ce moment ily a le patron du magasin}




\section{$\underline{\boldsymbol{o} \text { était en train de transporter... les produits }}$}

L'omission du pronom relatif n'étant pas possible en français L1, une telle formulation chez les apprenants sinophones relève d'une erreur d'ordre syntaxique. La source de cette différence est sûrement liée à la forme des structures présentatives en AVOIR de leur langue source ${ }^{10}$, puisque le chinois L1 présente le plus souvent la séquence yǒu $+\mathrm{SN}+\mathrm{SV}$, comme dans l'exemple (27). En effet, dans notre corpus des sinophones natifs 21 exemples sur 26 occurrences de structures présentatives en AVOIR totales ont cette forme.

\section{$\mathrm{CH} 1$ 同时间有一个警察也到了 \\ Tóng shijiān yǒu yí-ge jǐngchá yě dào-le \\ Même temps AVOIR un-CL policier aussi arriver-PFV \\ 'En même temps il y a un policier aussi [qui] est arrivé'}

En deuxième lieu, nous relevons chez les apprenants deux cas de structure en il y a comportant l'insertion d'un pronom personnel à l'emplacement qui serait réservé au pronom relatif qui en français L1 (exemple 28).

\section{FR2 il y avait un boulanger il décharge les pains}

L'insertion pronominale est également possible chez les francophones natifs, toutefois ce phénomène n'est pas attesté dans notre corpus en ce qui concerne ces derniers. En revanche, une telle configuration se trouve dans les productions des sinophones natifs (exemple 8 plus haut). En effet, comme nous l'avons vu (section 3.1) l'insertion d'un pronom personnel dans ce contexte sert à marquer une structuration explicite de l'information, notamment car l'expression du pronom sujet n'est pas grammaticalement obligatoire en chinois. En étudiant la structure correspondante en français L1, dans laquelle la proposition initiale, introduite par le présentatif il y a est suivie d'une proposition régie par un élément de reprise autre que le pronom relatif qui (exemple 29), certains auteurs ont remarqué qu'il s'agit d'une tournure que l'on trouve très fréquemment dans les productions enfantines et chez les porteurs d'un trouble du langage, sujets chez lesquels l'organisation du discours tend à expliciter des relations découlant directement de la structure informationnelle (Savelli et al. 2000 : 57 ; Hickmann 2003 ; Jullien 2005).

FR1 et ensuite il y a le chien il chasse le le chat (Hickmann 2003 : 249)

En général, ce type d'organisation, guidée par un principe pragmatique, se rapproche sensiblement du système du chinois, langue considérée par Li et Thompson (1976:457) comme «Topic-prominent ». Ainsi, lorsque les apprenants sinophones du français s'appuient sur ce type de structure pour introduire un référent nouveau, ils suivent probablement les mêmes principes de structuration de l'information que ceux dont ils disposent dans leur langue source.

En somme, lorsque les apprenants sinophones adoptent les structures en AVOIR pour présenter un nouveau référent dans leurs narrations en français L2, ils auront tendance à utiliser davantage les structures il y $a+\mathrm{SN}$ sans pseudo-relative (structure existentielle «simple »), ce qui n'est pas une erreur en soi mais contraste avec les productions des francophones natifs. Lorsque les sinophones utilisent la structure en AVOIR complexe en français L2, nous relevons l'omission du pronom relatif ainsi que l'insertion d'un pronom personnel à sa place, phénomènes qui reflètent une influence de leur langue source au niveau de la structure syntaxique, et de l'organisation pragmatique de l'énoncé.

\footnotetext{
${ }^{10} \mathrm{Je}$ remercie un relecteur anonyme d'avoir fait remarquer ce point
} 


\subsection{Les autres structures syntaxiques associées à la fonction d'introduction référentielle chez les apprenants sinophones}

\subsubsection{L'ordre verbe-sujet chez les apprenants sinophones}

Comme nous l'avons vu (section 3.2.2), les locuteurs sinophones natifs se servent parfois de l'ordre V-S ( 8 occurrences totales dans le corpus) pour introduire une nouvelle entité référentielle dans le discours (exemples 30a et 30b). Les francophones peuvent également y recourir dans les mêmes contextes ( 6 occurrences en français L1 dans notre corpus), comme montré dans les exemples en (31).

(30) a. CH1 这时候也出现了口个警察

Zhè shíhòu yě chūxiàn-le yí-ge jǔngchá

Ce moment aussi apparaître-PFV un-CL policier

Lit: 'A ce moment-là apparut aussi un policier'

b. $\mathrm{CH} 1$ 这个时候正好路边停了一辆车

Zhè-ge shíhòu zhènghăo lù=bian tíng-le yí-liang chē

$\mathrm{Ce}-\mathrm{CL}$ moment juste rue=sur arrêter-PFV un-CL voiture

Lit : 'Juste à ce moment au bord de la rue s'arrêta une voiture'

(31) a. FR1 Arrive un policeman, ils discutent

b. FR1 De l'autre côté de la rue vient Charlot qui marche (=19)

Chez les apprenants, nous ne relevons aucune occurrence de sujet postverbal dans le corpus ${ }^{11}$. Même dans les cas de verbes de déplacement comportant un sujet indéfini, le contexte «typique » pour une postposition du sujet en chinois L1, ces locuteurs restaurent l'ordre canonique S-V en français L2 :

\section{(32) a. FR2 Et à ce moment-là un policier est venu \\ b. FR2 et une autre dame est venue derrière elle \\ c. FR2 Et en ce moment-là un policier vient vers eux}

Ainsi, les apprenants sinophones ne produisent pas d'ordre V-S en français L2, alors que cet ordre est souvent employé dans des énoncés à nature thétique en chinois L1 et qu'il est attesté chez les francophones - dans notre petit corpus au moins.

Des études portant sur les productions d'apprenants sinophones peu avancés de l'italien (Valentini 1992 ; Chini 2002) ont remarqué que ces locuteurs produisent très tôt l'ordre V-S en L2, probablement favorisés par la disponibilité d'un tel arrangement des mots dans leur langue source (voir l'Introduction). Or en contraste de ces données il est intéressant de constater que dans notre corpus des apprenants sinophones, l'ordre V-S n'est jamais employé. Pourquoi ces locuteurs utilisent-ils systématiquement l'ordre $\mathrm{S}-\mathrm{V}$, tandis que dans leur langue maternelle l'inversion est tout aussi fréquente qu'en français? Nous avançons l'hypothèse que si nos participants sinophones, apprenants avancés de français L2, ne

\footnotetext{
${ }^{11}$ Nous avons chez les apprenants un seul cas de structure « à double sujet » où le sujet nominal apparaît en position postverbale au moyen du pronom explétif $i l$, dans une structure restrictive :
}

(II) FR2 Tout le monde s'en va il ne reste que la jeune fille qui reste là

En outre il ne s'agit pas de la première expression du référent de la « voleuse » donc cet exemple n'est pas compté dans les statistiques. 
reproduisent pas d'ordre V-S dans la langue cible, c'est que cette formulation n'est pas perçue comme un choix possible dans l'inventaire des tournures syntaxiques du français.

En effet, comme nous l'avons mentionné plus haut (section 3.2.2), si la configuration syntaxique à ordre verbe-sujet est possible et en chinois et en français L1, elle présente une différence quant au registre langagier et à la fréquence d'usage. En chinois l'ordre V-S est très fréquent aussi bien à l'écrit que dans des narrations orales ayant pour fonction de présenter des entités dans le discours, notamment avec des verbes de déplacements et ceux dénotant «l'apparition ». En revanche, l'ordre V-S en français relève d'un registre plus soutenu et n'est pas très fréquemment utilisé à l'oral. En même temps, l'ordre V-S en général est une structure acquise tardivement par les apprenants L2, par rapport aux structures $\mathrm{S}-\mathrm{V}$, et notamment dans la production ${ }^{12}$.

Ainsi la conceptualisation de la langue cible semble avoir également de l'influence dans le processus d'apprentissage. Notamment, elle peut freiner l'usage approprié d'une structure syntaxique - en l'occurrence l'ordre V-S - si la forme en question n'est pas répertoriée en tant que configuration alternative à l'ordre des mots « canonique » de la langue cible, et cela en dépit du fait qu'elle peut s'utiliser dans les mêmes contextes qu'en langue maternelle.

\subsubsection{Présentatives de PERCEPTION chez les apprenants sinophones}

Nous relevons dans le corpus des apprenants 9 cas de présentatives de PERCEPTION, assez fréquentes chez les francophones natifs ( $17 \%$ des énoncés incluant un nouveau référent) et peu employées dans les narrations en chinois L1 (5\%). Les apprenants ont moins systématiquement recours à des formules caractéristiques des productions des natifs telles que « on voit» (deux cas seulement) et nous n'avons relevé aucune occurrence de « on peut voir ». En revanche, dans leurs récits en français L2 les locuteurs sinophones se servent de tournures diversifiées : le locuteur est parfois le point de repère, le verbe voir étant donc décliné à la première personne (exemple 33a), ou bien ils s'appuient sur la perspective d'un autre personnage de la trame (exemple $33 \mathrm{~b}$ ).

\section{(33) a. FR2 j’ai vu une fille en passant la boulangerie ${ }^{13}$ \\ b. $\quad$ FR2 En voyant Chaplin qui s'approche...}

Nous incluons les exemples tel que (33b) dans la catégorie des présentatives de PERCEPTION, même donc lorsque le SN est introduit en référence à un autre personnage de la narration. Nous pensons que, comme les autres constructions présentatives, ces structures permettent aux locuteurs d'ancrer le référent visé dans le discours, dans ce cas au travers d'un repérage par rapport à la situation décrite. Lorsqu'un référent est présenté au moyen d'un verbe de PERCEPTION chez les apprenants, seuls deux exemples comportent une subordonnée relative, fait qui contraste nettement avec les productions en français L1 (34).

FR1 on peut voir une jeune fille qui se promène dans la rue, et puis... qui arrive devant une boulangerie

\footnotetext{
12 Je remercie un relecteur anonyme pour ses remarques qui m'ont permis de mieux développer ce point.

${ }^{13}$ Nous considérons cet exemple comme une structure présentative où le participe présent est improprement employé. Ce type de production pourrait s'inscrire dans la tendance générale relevée chez les apprenants d'éviter la reprise du SN par une subordonnée relative.
} 
Nous relevons quelques cas de présentatives de PERCEPTION associées à la fonction de réintroduction d'un référent. Nous aborderons l'encodage associé à ce type de statut référentiel du $\mathrm{SN}$ dans la section 4.4.

\subsubsection{Structures en c'est « à focus large » chez les apprenants sinophones}

Les structures en c'est «à focus large » (3.2.2) sont très peu employées par les apprenants sinophones (seulement 2 occurrences dans tout le corpus). De plus, tandis qu'en français L1 le SN est en même temps repris par le pronom relatif(exemple 35a), chez les apprenants c'est fonctionne en tant que prédicat unique (35b).

\section{(35) a. FR1 Donc, c'est une.. une fille qui regarde des pâtisseries en vitrine \\ b. FR2 en face d'elle c'est une boutique}

En outre, nous ne relevons pas chez les apprenants de structures en c'est «à enchainement» (du type «c'est alors que... »). Les structures en c'est à focus large sont employées par les apprenants sinophones pour introduire des entités référentielles inanimées; dans un même temps, une fois de plus, ces locuteurs tendent à éviter la reprise relative qui est un trait caractéristique des tournures déployées en français L1. Cela se différencie également des constructions équivalentes en chinois L1, qui comportent souvent une suite de deux prédicats (3.2.2). Ainsi, les apprenants utilisent très rarement l'introducteur c'est pour ancrer un nouveau référent dans la narration et ils évitent notamment la continuation relative portant sur le $\mathrm{SN}$, de plus aucune occurrence de structure en c'est « à enchainement » n'est relevée dans leur corpus. Il est intéressant de constater que, d'autre part, tous les apprenants sinophones maîtrisent correctement la forme en c'est...qui à fonction identificatrice en français L2, où le SN dénote un référent focalisé en contraste à un ensemble de référents possibles :

\section{FR2 le monsieur a dit que c'est lui qui a piqué la baguette \\ FR2 ce n'est pas la fille qui a volé le pain c'est lui-même qui a volé}

Dans ce contexte, les apprenants n'utilisent jamais l'ordre non marqué S-V et nous ne relevons aucune erreur dans leurs productions : le SN focalisé est toujours repris par une subordonnée relative ( «c'est SN qui ... ») et ni l'omission du pronom relatif ni l'insertion d'un pronom personnel ne sont pratiquées, contrairement à ce qui caractérise les structures «présentatives » en AVOIR chez les apprenants (section 4.2).

Le fait que les locuteurs sinophones n'utilisent pas improprement d'ordre non marqué S$\mathrm{V}$ dans ce contexte est probablement favorisé par la correspondance étroite en français entre la forme de la structure en c'est...qui et l'expression d'une proposition à focus étroit, contrairement aux structures «présentatives », lesquelles commutent à priori avec l'ordre non marqué S-V. En théorie, il n'y a pas de correspondance unique entre la forme d'une « structure présentative » et la fonction d' «introduction référentielle » en français - ni en chinois d'ailleurs. Cela est témoigné par la présence dans notre corpus des natifs d'un nombre non négligeables d'occurrences ( $29 \%$ chez les francophones et $34 \%$ chez les sinophones) où 
l'introduction d'un nouveau référent est réalisée dans l'ordre non marqué $\mathrm{S}-\mathrm{V}$, le SN visé apparaissant en position préverbale « canonique ${ }^{14}$.

En revanche, la structure c'est...qui est prototypiquement associée à l'expression du focus contrastif en français (Lambrecht 2001), où l'élément clivé est mis en avant en contraste à un ensemble d'éléments possibles, et d'autre part cette forme trouve son correspondant dans la configuration syntaxique du chinois en "shi...de " ('être...SUB'), qui s'emploie dans les mêmes contextes :

\section{$\mathrm{CH} 1$ 不是这位女孩偷的, 是我偷的 \\ Bu shì zhè-wei nühái tōu de, shì wǒ tōu de NEG être ce-CL fille voler DE être $1 \mathrm{~S}$ voler SUB 'Ce n'est pas cette fille [qui] a volé, c'est moi [qui] ai volé'}

La présence du marqueur de subordination nominale de 的 au sein de la construction à focus étroit en chinois L1 pourrait favoriser l'apparition d'une subordonnée en qui dans les productions des apprenants sinophones en français L $2^{15}$. En même temps, nous constatons que la fréquente omission de la proposition relative relevée chez les apprenants est un phénomène qui affecte principalement les structures «présentatives» tandis qu'elle ne concerne pas les clivées à focus étroit en c'est.

Bartning et Kirchmeyer (2003) remarquent que les relatives « continuatives » (exprimant des informations qui font avancer le récit) sont apprises tardivement par rapport aux relatives «descriptives » (celles qui encodent des informations d'arrière-plan). En effet, dans leurs récits les apprenants sinophones utilisent systématiquement des relatives exprimant des informations déjà connues :

(39) a. FR2 le monsieur qui était par terre il a-il voulait sauver la vie de la fille

\section{b. FR2 Et cette fille qui a piqué la baguette s'est cognée sur un monsieur}

La proposition relative dans la structure en c'est...qui à focus étroit - contrairement à la même structure lorsqu'elle exprime une articulation focale entièrement rhématique - encode typiquement de l'information d'arrière-plan, tandis que le SN clivé dénote l'élément focalisé.

En résumé, l'emploi très rare dans le corpus des apprenants sinophones des structures en c'est...qui à focus large peut refléter une tendance à l' « unicité des fonctions » (Bartning et Kirchmeyer 2003), dans la mesure où la structure en c'est...qui est premièrement associée par les apprenants à un autre type d'articulation focale, à savoir l'expression du focus contrastif. D'autre part, les sinophones pourraient également être guidés ici par leur langue source : lorsqu'ils ont recours à une construction présentative, les structures en AVOIR sont préférées, à l'instar du chinois L1 qui présente un inventaire moins diversifié d'introducteurs faisant premièrement appel aux structures en yŏu 'avoir' pour assurer la fonction d'introduction référentielle.

\subsection{Structures syntaxiques liées à la fonction de réintroduction référentielle}

Chez les francophones natifs, la structure présentative en AVOIR (40a), ainsi que, même s'il s'avère minoritaire, l'ordre V-S (40b), peuvent s'utiliser également pour encoder la

\footnotetext{
${ }^{14}$ La nature de l'expérience linguistique peut influencer les résultats en cet égard, dans la mesure où les participants adoptent parfois un ton plus descriptif et produisent une narration de type « compte-rendu » que l'on n'observerait pas dans la conversation spontanée (informelle).

${ }^{15}$ Remarquons toutefois qu'en chinois le syntagme verbal précède la particule DE selon la structure shì SN V DE.
} 
fonction de réintroduction référentielle. Un référent « réintroduit » est un référent qui a été actif dans l'esprit des interlocuteurs dans un segment du discours précédent, mais ne l'est plus au moment de l'énonciation de la phrase : il fait partie de ces entités référentielles « accessibles » grâce au contexte linguistique (Lambrecht 1994 : 100). La séquence où la voleuse attend Charlie Chaplin près du commissariat nous permet d'observer comment les locuteurs gèrent la reprise d'un participant qui revient sur la scène suite à une interruption narrative.

\section{(40) a. FR1 il y a la fille qui l'attend à la sortie \\ b. FR1 et là au coin de la rue l'attend la femme}

Contrairement aux francophones (40), les apprenants sinophones n'utilisent pas de structures présentatives dans ce contexte. Tout comme pour ce qui est de la fonction d'introduction référentielle, les énoncés à ordre V-S ne sont pas employés pour réintroduire un référent dans le discours narratif. La présence d'un verbe inaccusatif, tel que apparaître dans les exemples en (41), ne bloque pas non plus dans ce cas l'encodage par un ordre S-V :

\section{(41) a. FR2 ce jeune fille est apparu \\ b. FR2 il a l'air de chercher quelqu'un et après la fille... la fille apparaît}

D'autre part, dans le contexte de référents réintroduits, nous ne constatons non plus de cas de structures centrées sur le verbe AVOIR. A première vue, l'on pourrait supposer que les apprenants transposent dans la langue cible la restriction de définitude qui caractérise les tournures en yǒu - ainsi que l'ordre V-S - du chinois L1, car ces structures enchâssent très difficilement des SN définis (section 3.1). Toutefois, nous relevons chez les apprenants des cas où le SN apparaissant au sein de la structure en il y a est défini (exemples 42a et 42b). Par conséquent, il ne s'agit pas d'une contrainte d'ordre morphosyntaxique :

\section{(42) a. FR2 il y avait le personnel de la boulangerie qui vient de sortir quelque chose d'un-d'une grande voiture \\ b. FR2 Pendant ce moment il y a le patron du magasin était en train de transporter les produits $\quad(=26 \mathrm{~b})$}

Rappelons ici la distinction entre le statut cognitif des référents et leur manifestation linguistique, qui relèvent de catégories distinctes : l'une cognitive et l'autre formelle. Ainsi, la corrélation entre l' « identifiabilité » (cognitive) et la « détermination » (grammaticale) est imparfaite car certains référents non identifiables sont codés par des SN définis, et vice-versa (Lambrecht 1994 : 105). Cela s'explique par le fait qu'à une catégorie formelle, discrète, correspond une catégorie cognitive non discrète (Lambrecht 1994 : 84).

Dans les exemples ci-dessus, les SN définis «le personnel de la boulangerie » et «le patron du magasin » dénotent des référents non mentionnés auparavant dans le discours : lorsque les structures présentatives en AVOIR chez les apprenants sinophones comportent un SN défini, il s'agit toujours d'énoncés encodant la première expression du référent visé. Ces données nous montrent que si les apprenants se conforment à la forme des structures présentatives de la langue cible, ils ne parviennent pas à lui confier une fonction dont la structure de la langue source est dépourvue, en l'occurrence l'expression d'entités « nouvelles » sur le plan relationnel mais « déjà évoquées » quant à leur statut référentiel (section 2.1). 
Les résultats préliminaires d'une expérience parallèle que nous avons conduite sur des apprenants francophones de chinois L2 semblent conforter cette hypothèse car, à l'inverse, ces sujets se servent systématiquement des structures présentatives en yǒu, jusqu'à les employer même là où leur usage est pragmatiquement inapproprié, notamment en présence de référents réapparaissant dans la scène du discours (exemple 43). Dans ce cas, les apprenants ne parviennent pas à dissocier de la forme syntaxique en question une fonction qui est présente dans leur langue source, à savoir l'expression des référents réactivés.

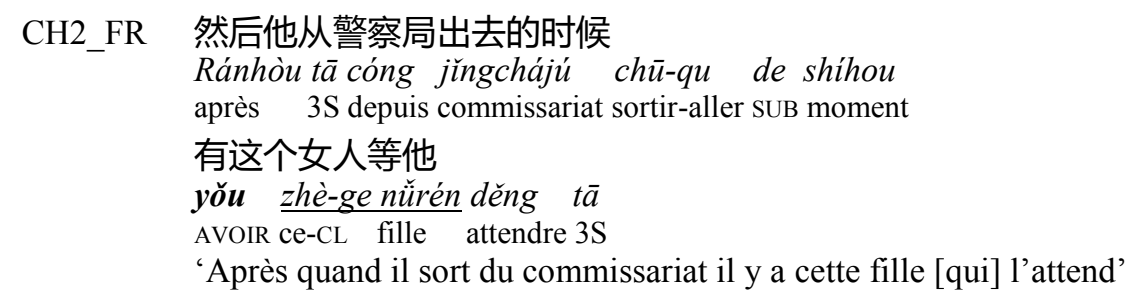

D'autre part, chez les apprenants sinophones les référents de type «réintroduit » sont codés sans déploiement d'un marquage syntaxique particulier, exception faite de quatre occurrences comportant un verbe de PERCEPTION, toutes construites avec une proposition complétive (la forme voir que $S N$... n'étant pas une "construction présentative» à proprement parler) :

\section{FR2 Et on voit que la jeune dame est juste cachée au coin de la rue}

En revanche, les apprenants ont tendance à marquer le SN dénotant ces référents au moyen d'un adjectif démonstratif (45a), ou par une dislocation (45b; introduite par une spécification progressive en $45 \mathrm{c}$ ), tournures que l'on n'aura pas relevées en français L1 dans ce contexte :

(45) a. FR2 lorsque ce garçon est sorti de la préfecture...

b. FR2 la fille elle l'a attendu dehors

c. FR2 l'homme, le héros il est sorti de la police

Les différents procédés peuvent coexister dans le même énoncé. Dans l'exemple (46), le locuteur sinophone désigne le référent de la «voleuse » par un pronom démonstratif, une reprise pronominale en tant que complément d'objet direct du verbe de perception voir et ensuite comme pronom sujet de troisième personne (le pronom il est improprement utilisé car en chinois oral le pronom de troisième personne $t \bar{a}$ ne varie pas par rapport au genre). En outre, nous observons que les apprenants recourent également à des stratégies de "scene setting », qui préparent la réintroduction du référent visé (47) :

\section{FR2 Et cette femme je la vois aussi il attend le monsieur secrètement près de la police \\ FR2 Et à la scène suivante on voit la porte du commissariat. Il y a un policier qui a ouvert la porte et Chaplin sort de la porte}

D'autre part, on remarquera que dans le corpus des sinophones natifs, les référents réintroduits sont systématiquement marqués par l'emploi d'un démonstratif et d'une dislocation (48) :

(48) a. CH1 这个口 प 她就在外面等他 
Zhè̀-ge nǘshēng $\boldsymbol{t} \overline{\boldsymbol{a}}$ jiù zài wàimiàn děng tā $\mathrm{Ce}-\mathrm{CL}$ fille $3 \mathrm{~S}$ donc se.trouver dehors attendre $3 \mathrm{~S}$ Lit: 'Cette fille elle l'attendait dehors'

\section{b. $\mathrm{CH} 1$ 然后刚刚被逮捕的这个人他出来了}

Ránhòu gānggāng bèi dàibǔ de zhè-ge rén tā chū-lái-le

Après juste PASS arrêter SUB ce-CL personne 3S sortir-venir-PFV

Lit: 'Après cet homme qui venait de se faire arrêter il est sorti'

En résumé, nous constatons que les locuteurs sinophones de français L2 n'utilisent jamais de structures présentatives en AVOIR ni d'ordre V-S pour marquer les référents réactivés mais qu'en revanche ils s'appuient sur des procédés divers - notamment le marquage du SN par un adjectif démonstratif et la dislocation - calqués sur ceux employés dans leur langue source.

\subsection{Reprise du SN (ré)introduit par les structures «introducteur + SN » : confrontation entre français L1 et L2}

Nous avons vu dans la section 4.2 que les apprenants utilisent le plus souvent les structures en AVOIR « simples » au détriment des « complexes » et qu'ils produisent des exemples sans le pronom relatif qui. Ces faits se relient à un phénomène plus général relevé chez les apprenants, à savoir la tendance à éviter la reprise du SN dénotant le référent visé ( « introduit » ou bien « réintroduit ») faite au moyen d'une relative. En effet, on remarquera que cette tendance en français L2 ne concerne pas seulement les phrases présentatives en AVOIR mais aussi les autres constructions syntaxiques ayant la même fonction discursive et qui se construisent toutes, en français L1, avec une proposition relative (à savoir les présentatives de PERCEPTION, les structures en c'est à focus large mais aussi l'ordre V-S parfois ; section 3.2). Ainsi, dans le tableau suivant nous présentons un aperçu général de la fréquence absolue et relative des diverses structures syntaxiques ayant la fonction d'introduction et de réintroduction d'un référent dans la narration, classées ici en fonction des éléments qui suivent le SN focalisé ${ }^{16}$.

Tableau 2. Reprise du SN (ré)introduit par les structures « introducteur (INTRO) + SN » dans les récits des natifs (FRL1) et des apprenants sinophones (FRL2)

\begin{tabular}{|c|c|c|c|c|}
\hline Groupes de locuteurs & \multicolumn{2}{|c|}{ FRL1 } & \multicolumn{2}{|c|}{ FRL2 } \\
\hline Nombre d'énoncés total & \multicolumn{2}{|c|}{589} & \multicolumn{2}{|c|}{652} \\
\hline Occurrence absolue (N) et relative (\%) & $\mathrm{N}$ & $\%$ & $\mathrm{~N}$ & $\%$ \\
\hline $\begin{array}{l}\text { [INTRO + SN1] | SN2 ... } \\
\text { «Dans la vitrine il y a des pâtisseries } \mid \text { Elle a l'air hésitant » }\end{array}$ & 4 & 0.6 & 12 & 1.8 \\
\hline $\begin{array}{l}{[\mathrm{INTRO}+\mathrm{SN}] \text { qui ... }} \\
\text { «On voit Charlot qui sort » }\end{array}$ & 38 & 6.4 & 7 & 1 \\
\hline $\begin{array}{l}{[\text { INTRO }+\mathrm{SN}] \varnothing \mathrm{V}} \\
\text { «Il y avait une femme plus âgée est arrivée » }\end{array}$ & -- & 0 & 3 & 0.4 \\
\hline $\begin{array}{l}\text { [INTRO + SN] SNPRO } \\
\text { «Il y } a \text { une fille sans domicile. Elle a faim » }\end{array}$ & 3 & 0.5 & 10 & 1.5 \\
\hline SOUS-TOTAL & 45 & 7.6 & 32 & 4.9 \\
\hline
\end{tabular}

\footnotetext{
${ }^{16}$ La classification proposée dans le Tableau 5 ne comprend pas les complétives (« et on voit que la jeune dame est juste cachée au coin de la rue ») ni les structures en c'est " à enchainement » (" c'est là que le boulanger arrive »), ces dernières relevées seulement chez les francophones d'ailleurs (section 4.3.2)
} 
La première séquence [INTRO + SN1] $\mid$ SN2 décrit toute occurrence de structure syntaxique marquée du type « introducteur $+\mathrm{SN}$ » où le référent (ré)introduit n'est pas repris dans le contexte de droite, en présence donc d'une transition référentielle. Cela comprend la catégorie des phrases existentielles « simples » à l'exclusion des énoncés donnant suite à une reprise pronominale. Il s'agit de la tournure la plus fréquente en français L2 :

(49) FR2 et entour de ces trois uns il y a un police et beaucoup de personnes passa-passantes | Et monsieur Chaplin (...)

Dans une portion importante des résultats, les locuteurs sinophones utilisent un « introducteur » pour ancrer un référent dans la narration de manière accessoire, sans que son maintien soit assuré dans le discours immédiat. Cela reflète une tendance plus générale relevée dans l'organisation discursive chez les apprenants, notamment le recours à de fréquentes transitions de topiques et l'organisation du récit en chaînes référentielles plus brèves par rapport aux productions des locuteurs natifs (Hendriks 2003 ; Ryan 2015).

La deuxième séquence [INTRO $+\mathrm{SN}$ ] $+q u i \mathrm{~V}$ inclut tous les exemples de structure présentative où le SN est repris par une proposition relative. Il s'agit manifestement de la tournure la plus utilisée par les francophones natifs (38 occurrences sur 45$)^{17}$. Dans le corpus des apprenants, cette forme est utilisée mais reste minoritaire ( 7 occurrences) :

FR2 en arrière elle voit un boulanger qui vient de sortir des pains d'un camion

La troisième séquence $[$ INTRO $+\mathrm{SN}] \varnothing \mathrm{V}$ reflète les erreurs relevées seulement chez les apprenants sinophones qui comportent l'omission du pronom relatif qui (section 4.2). Ce phénomène ne concerne que leurs structures présentatives en AVOIR :

FR2 et derrière elle il y avait une femme plus âgée est arrivée

La dernière séquence [INTRO $+\mathrm{SN}] \mathrm{SN}_{\mathrm{PRO}}$, où le $\mathrm{SN}$ est encodé par un pronom personnel au lieu d'un relatif, regroupe soit les deux occurrences de il y a SN il(s)... discutées dans la section 3.1, comportant une insertion pronominale au sein de la structure présentative (dans lesquelles la courbe prosodique nous indique qu'il s'agit sans doute d'une structure unitaire), ainsi que les énoncés tels que les exemples en (52) où la reprise pronominale se fait dans le segment de texte suivant. Une telle structuration de l'information est très rare dans les productions des francophones, en revanche chez les apprenants elle est la plus fréquemment utilisée lorsque le référent dénoté par le SN est maintenu dans le contexte de droite, autant dans le cas de structures en AVOIR (52a) qu'en présence d'autres introducteurs (52b) :

(52) a. FR2 et derrière cette fille il y a de femme très élégante et un peu âgée elle trouve que cette fille a volé quelque chose $e^{18}$

b. FR2 Tout d'abord je vois une fille avec les pieds nus il a l'air.. l'air comment dire pauvre

En somme, chez les francophones natifs le SN focalisé est repris par un pronom relatif dans la grande majorité des cas. En général, le maintien référentiel fait au moyen d'une

\footnotetext{
${ }^{17}$ Nous incluons les cas d'ordre V-S suivis par une reprise relative :

(III) FR1 et là, au coin de la rue l'attend la femme, la pauvre femme, qui en le voyant lui saute au cou de joie

${ }^{18} \mathrm{Je}$ remercie un lecteur anonyme de m'avoir fait noter que cet exemple est mieux analysé comme une phrase existentielle « simple » à part entière ( « derrière cette fille il y a de femme très élégante et un peu âgée ») suivie par une reprise pronominale (" elle trouve que... »).
} 
subordonnée relative est un procédé fréquemment employé par les locuteurs francophones (Hendriks $2003: 300)$ :

FR1 donc et on voit la personne ou le boulanger
on sait pas trop qui c'est
qui.. qui descend qui descend les les-
qui prend les pains ou les pâtisseries dans le camion
qui est stationné devant la boulangerie
et puis qui rentre dans la boulangerie

Tandis que les natifs sont capables d'articuler leur discours en alternant relatives « descriptives » et « continuatives », pour faire avancer la trame et apporter des informations déjà connues, tout en maintenant la continuité référentielle (53), une telle planification du discours s'avère difficile à maîtriser par les apprenants. Chez ces locuteurs, lorsqu'un référent est maintenu au rang de topique de la narration, le marquage de la continuité référentielle se fait le plus souvent sans le recours à une proposition relative :

\author{
FR2 Il y a une jolie fille très.. très pauvre, \\ parce qu'elle est mal habillée et avec pieds nus, \\ elle a très très faim \\ du coup elle a arrêté devant un boulangerie
}

L'emploi d'un pronom personnel pour assurer le maintien référentiel ne semble pas refléter dans ce cas la volonté de lever une possible ambiguïté. Ryan (2015) suggère que les locuteurs sinophones «ne feraient pas confiance » aux pronoms personnels de troisième personne dans la L2, puisque ceux-ci ne portent pas de distinction sur le genre en chinois L1 (oral). En effet, si le pronom personnel a l'avantage d'indiquer le genre de son référent contrairement au pronom relatif qui, paradoxalement son emploi peut conduire l'apprenant sinophone à un usage inapproprié :

\title{
FR2 Un jour au vitrine d'une boulangerie il y a une jeune fille, il a faim mais il n'a pas d'argent
}

Lorsque les apprenants assurent le maintien d'un référent au travers d'un pronom personnel, ils semblent recourir davantage à des moyens équivalents à ceux de leur langue source dans le processus général de la construction du discours, au lieu de se rapprocher des stratégies adoptées en français L2, à savoir la succession de plusieurs propositions relatives portant sur le référent dont il est question.

Ainsi, en examinant le contexte d'occurrence des structures «présentatives", nous constatons qu'au-delà de l'empaquetage de l'information au niveau phrastique, les productions des apprenants sinophones se distinguent clairement de celles des francophones natifs quant à la structuration globale de leur récit.

\section{Conclusions}

Dans ce travail, nous avons tout d'abord analysé l'expression des nouvelles entités référentielles à l'œuvre chez les apprenants sinophones du français. Notre étude prenait comme point de départ le constat que le chinois et le français L1 disposent tous deux d'une tournure présentative s'appuyant sur le verbe AVOIR, présentant des convergences formelles et fonctionnelles non négligeables. Nous nous sommes proposée d'observer l'effet d'une telle proximité sur l'interlangue des apprenants.

Il ressort des données analysées ici que les structures présentatives en AVOIR sont effectivement la stratégie la plus fréquemment utilisée pour introduire une nouvelle entité 
référentielle en chinois et français L1. Les apprenants quant à eux se distinguent premièrement dans leur propension très inégale à recourir à ce type de marquage : en dépit de l'affinité entre la langue maternelle et la langue cible, ces locuteurs privilégient l'ordre sujet-verbe en français L2.

Si l'on examine dans les détails la première expression de l'un des référents, notamment celui de la dame «moucharde » (section 4.1.2), cette divergence devient frappante : partagés entre deux pôles, avec d'un côté des productions en chinois L1 qui s'appuient largement sur la construction passive, et de l'autre des francophones natifs, chez lesquels l'introduction de ce référent est systématiquement réalisée par la construction présentative en AVOIR, les apprenants se distinguent en rétablissant le plus souvent l'ordre non marqué S-V.

Nous reconnaissons en premier lieu une complexité structurelle des formes en il y a par rapport à celles en yǒu 'avoir' du chinois, les premières demandant une certaine planification, notamment la conjugaison du verbe avoir ainsi que son accord avec le deuxième prédicat. De là viendrait aussi la préférence chez les apprenants pour les phrases existentielles « simples ", dépourvues de proposition relative. D'autre part, lorsque ces locuteurs produisent des structures en AVOIR « complexes » comportant l'omission du pronom relatif qui, ils calquent probablement l'arrangement syntaxique dont ils disposent dans leur L1. Une formulation syntaxique relevée dans les corpus des apprenants comporte un pronom personnel à la place du pronom relatif; dans ce cas nous avons probablement affaire à un transfert de la langue source, laquelle présente ce modèle, mais également à un traitement de l'information de type «topique-commentaire » qui, plus généralement, reflète la structuration du discours à l'œuvre en chinois L1 (section 4.2).

Enfin, les apprenants n'emploient pas de constructions en AVOIR pour dénoter des référents « réintroduits » en français $\mathrm{L} 2$, et ils ne confient donc pas à cette structure une fonction qui n'est pas présente dans l'éventail de fonctions associées à la forme correspondante de leur langue source. Notamment, les locuteurs sinophones s'adaptent aux différences de type formel de la langue cible (SN défini) mais non à celles d'ordre pragmatique (référent réactivé) ; consulter la section 4.4.

En général, les productions des apprenants sinophones en français L2 se distinguent clairement de celles des francophones natifs lorsqu'on prend en compte l'ensemble des structures syntaxiques associées à la (ré)introduction référentielle : en effet, ces derniers recourent à un inventaire plus dynamique et diversifié de structures présentatives, incluant également les présentatives de PERCEPTION et celles en c'est "à focus large », mais aussi quelques cas d'ordre V-S, et ces structures ont toutes en commun le fait de reprendre le SN visé par une subordonnée pseudo-relative régie par le pronom qui. De leur côté, les apprenants font davantage appel à des procédés analytiques, tels que la reprise pronominale explicite (section 4.5).

La tendance à structurer l'information de façon analytique est un trait caractéristique des productions des apprenants, lesquels même lorsqu'ils atteignent un niveau avancé de la langue cible ne parviennent pas à rejoindre les productions des natifs qui, eux, sont capables de synthétiser et de condenser la structure informative de leur discours : « la structuration du récit est plus linéaire et analytique chez les apprenants que chez les natifs, qui synthétisent plusieurs événements dans un seul énoncé en hiérarchisant et nuançant l'information » (Bartening et Kirchmeyer 2003).

A cet égard, on remarquera que si chez les apprenants francophones de l'italien ou de l'anglais L2, cette tendance se manifeste dans un usage systématique et parfois redondant des structures "présentatives » dans la langue cible (Turco 2008 ; Lambert 1998 ; Leclercq 2008 ; voir l'Introduction), manifestement cela n'est pas le cas des apprenants sinophones. Chez ces locuteurs, les tournures en il y $a$ du français L2 ne sont pas utilisées à l'instar des structures en yǒu, stratégies syntaxiques pourtant très couramment employées dans les narrations orales en chinois L1 et qui permettent notamment l'ancrage des entités dans le 
discours. Nous pensons que le facteur déterminant ici est la reprise relative en français, laquelle est absente des structures " présentatives » au sein de la langue source et qui, plus en général, n'est pas en chinois un moyen utilisé pour structurer globalement le discours autour d'un référent. Ainsi, lorsque les apprenants sinophones de français L2 marquent la continuité référentielle vis-à-vis d'une entité introduite par une structure syntaxique marquée, ils auront plus souvent recours à une formulation encore plus « analytique », notamment la reprise explicite par un pronom de personne (ou bien un SN lexical) et non pas l'enchaînement de propositions relatives portant sur le $\mathrm{SN}$, typique des récits des francophones natifs.

En conclusion, cette étude a montré que la présence d'une structure comparable dans la langue maternelle ne favorise pas un usage approprié en langue seconde, puisqu'en premier lieu les apprenants sinophones adoptent l'ordre non marqué pour introduire les entités référentielles dans leur discours en français L2. Une telle difficulté face à la maîtrise pragmatique de ces structures est sûrement influencée par le fait qu'elles n'ont pas une relation «exclusive» avec la fonction d'introduction référentielle, étant donné qu'effectivement elles commutent a priori avec l'ordre S-V. A titre de comparaison, nous avons remarqué que tous les apprenants sinophones qui ont participé à notre expérience maîtrisent correctement la structure c'est...qui à focus étroit, prototypiquement associée à la fonction d'identification référentielle en français et laquelle trouve son " homologue » dans la forme chinoise shi ... de. Dans ce cas les apprenants ne produisent pas improprement l'ordre non marqué S-V et n'omettent jamais la proposition relative introduite par qui. Le fait que cette structure présente le plus souvent une relative encodant de l'information déjà connue est également un facteur qui concourt à favoriser son apparition en français L2 (section 4.3.3).

D'autre part, nous n'avons pas relevé dans le corpus des apprenants sinophones de structures à ordre V-S, alors que cet ordre est souvent employé dans des énoncés à nature thétique en chinois L1 et qu'il est attesté dans le corpus des francophones natifs. Nous pensons que chez les apprenants sinophones l'emplacement postverbal du sujet n'est pas perçu comme une tournure possible en français et par conséquent l'ordre sujet-verbe est restauré.

Ainsi, si d'un côté les principes de structuration qui guident l'organisation de l'information dans la langue source jouent un rôle central dans la manière dont les apprenants analysent et organisent le contenu informatif de la langue cible, nous pensons que la conceptualisation de la L2 a également un certain poids : chez nos apprenants avancés, bénéficiant tous d'une connaissance métalinguistique poussée de la langue cible - dont d'ailleurs ils se servent surtout dans le milieu académique, l'ordre linéaire sujet-verbe est perçu en tant qu'arrangement syntaxique « canonique » en français, et par conséquent c'est à cet ordre qu'ils font premièrement appel dans leurs productions en langue cible, ce qui est tout à fait «correct» du point de vue de la forme mais peut résulter en un choix pragmatiquement inapproprié, à des degrés différents selon le contexte énonciatif.

Les hypothèses avancées dans ce travail ne sont qu'un point de départ pour des recherches ultérieures basées sur un recueil de données plus large, visant à diversifier le profil sociolinguistique des locuteurs sinophones mais également dans le but de mettre en relation les résultats avec l'analyse des stratégies adoptées par des locuteurs de langues sources différentes dans les mêmes contextes discursifs.

\section{LISTE DES GLOSES UTILISEES DANS LES EXEMPLES}

$3 \mathrm{~S}$ pronom de troisième personne du singulier 
CL classificateur

SUB particule subordinative et possessive $d e$ 的

PROG adverbe marquant l'aspect progressif zai 在

$\mathrm{NE} \quad$ particule $n e$ 呢 marquant la pause

$\mathrm{PFV}$ particule marquant l'aspect perfectif

\section{Bibliographie}

Ashby, W. J. (1995). French presentational structures. In J. Amastae et al. (eds), Contemporary research in Romance Linguistics. Amsterdam: John Benjamin Publishing, 91104.

Ashby, W. J. (1999). Au sujet de quoi ? La fonction du sujet grammatical, du complément d'objet direct, et de la construction présentative en français parlé. French Review, 481-492.

Bartning, I. et Kirchmeyer, N. (2003). «Le développement de la compétence textuelle à travers les stades acquisitionnels en français L2 », Acquisition et interaction en langue étrangère, 19, en ligne : http://aile.revues.org/1112

Bentley, D., Ciconte, F. M., et Cruschina, S. (2015). Existentials and Locatives in Romance Dialects of Italy. Oxford: Oxford University Press.

Berrendonner, A. (2003). «Grammaire de l'écrit vs. grammaire de l'oral : le jeu des composantes micro- et macro-syntaxiques. » In Rabatel, A. Interactions orales et contexte didactique. Lyon : Presses Universitaires. 249-264

Blanche-Benveniste, C. (1983). Examen de la notion de subordination. Recherches sur le français parlé, 4, 71-115.

Blanche-Benveniste, C. (2000). Approches de la langue parlée en français. Paris: Ophrys.

Cài, W. (2000). Dài dìngzhǐ jiānyǔ de "yǒu" zìjù [Les phrases en yǒu comportant un pivot défini]. Journal of Zhenjiang teachers college (2).

Cappeau, P. et Deulofeu, J. (2001). Partition et topicalisation : il y en a « stabilisateur » de sujets et de topiques indéfinis. Cahiers de praxématique (37), 45-82.

Cappeau P. et Lahousse K. (2015). « Le sujet postposé », in Encyclopédie Grammaticale du Français, en ligne : http://encyclogram.fr.

Carroll, M. et Lambert, M. (2003). Information structure in narratives and the role of grammaticised knowledge: A study of adult French and German learners of English. Information structure and the dynamics of language acquisition, 267-287.

Carroll, M. et Lambert, M. (2006). Reorganizing principles of information structure in advanced L2s. In Byrnes, Weger-Guntharp et Sprang (eds. ) Educating for Advanced Foreign Language Capacities: Constructs, Curriculum, Instruction, Assessment, (pp. 54-73). Georgetown DC: University Press. 
Chao, Y. (1968). A grammar of spoken Chinese. Berkeley and Los Angeles: University of California Press.

Chang, H. (2004). Definite NPs in Mandarin you-existentials. The proceedings of the 40th Annual Meeting of the Chicago Linguistic Society (CLS-40), 1-11.

Chini, M. (2002). Ordres marqués et perspective du locuteur en italien L2. Revue française de linguistique appliquée, 7(2), 117-137.

Chini, M. et Lenart, E. (2008). Identifier le topique dans une tâche narrative en italien et en français chez les natifs (L1) et les apprenants (L2). Acquisition et interaction en langue étrangère, (26), 113-148.

Choi-Jonin, I. et Lagae, V. (2005). Il y a des gens ils ont mauvais caractère. À propos du rôle de il y a. In A. Murguía (éd.): Sens et Références. Tübingen : Gunter Narr Verlag, 39-66.

Cornish, F. (2005). Une approche pragmatico-discursive des phrases « thétiques ». Mélanges en l'honneur de Claude Muller: la syntaxe au cour de la grammaire. Rennes: Presses Universitaires de Rennes, 75-84.

Côté, M. (1998). Quantification over individuals and events and the syntax-semantics interface: the case of the existential constructions. In Kimary Shhin, Susan Blake et EunSook Kim (eds.) The proceedings of the 17th West Coast Conference on Formal Linguistics, 147-161.

Creissels, D. (à paraître). Existential predication in typological perspective. En ligne : www.deniscreissels.fr/public/Creissels-Exist.Pred.pdf.

Hancock, V., et Kirchmeyer, N. (2005). Discourse structuring in advanced L2 French: The relative clause. Focus on French as a foreign language, 17-35.

Heine, B. et Kuteva, T. (2005). Language contact and grammatical change. Cambridge: Cambridge University Press.

Hendriks, H. (2003). Using nouns for reference maintenance: A seeming contradiction in L2 discourse. In A. G. Ramat (Ed.), Typology and second language acquisition, 291- 326. Berlin: Mouton de Gruyter.

Hendriks, H. et Watorek, M. (2008). L'organisation de l'information en topique dans les discours, Acquisition et interaction en langue étrangère, 26, en ligne : http://aile.revues.org/3372

Hu, J. et Pan, H. (2008). Focus and the basic function of Chinese existential you-sentences. In Comorovski I., von Heusinger K. (eds) Existence: Semantics and Syntax. Studies in Linguistics and Philosophy, vol 84. (pp. 133-145) Dordrecht: Springer.

Huang, S. (2013). Chinese Grammar at Work (Vol. 1). Amsterdam: John Benjamins Publishing Company. 
Jeanjean, C. (1979). Soit y'avait le poisson soit y'avait ce rôti farci. Etude de la construction de il y a dans la syntaxe du français. Recherches sur le Français Parlé Aix-en-Provence, (2), 121-160.

Kail, M. (1991). Acquisition des langues premières et secondes dans une perspective interlangues. Revue française de pédagogie, 67-78.

Karssenberg, L. (à paraitre). French il ya clefts, existential sentences and the Focus-Marking Hypothesis. Journal of French Language Studies.

Karssenberg, L. et Lahousse, K. (à paraitre). The information structure of French il y a clefts et $c$ 'est clefts: a corpus-based analysis. Linguistics.

Klein, W. et Perdue, C. (1992). Why does the production of some learners not grammaticalize? Studies in Second Language Acquisition, (14) 259-272.

Kuroda, S. Y. (1972). The categorical and the thetic judgment: Evidence from Japanese syntax. Foundations of language, 153-185.

Lahousse, K. (2011). Quand passent les cigognes. Le sujet nominal postverbal en français moderne. Presses universitaires de Vincennes.

Lambert, M. ; Carroll, M. et von Stutterheim, C. (2008). Acquisition en L2 des principes d'organisation de récits spécifiques aux langues. Acquisition et interaction en langue étrangère (26), 5-10.

Lambrecht, K. (1987a). Aboutness as a Cognitive Category: The Thetic-Categorical Distinction Revisited. Annual Meeting of the Berkeley Linguistics Society (13), 366-382.

Lambrecht, K. (1987b). Presentational cleft constructions in spoken French. In John Haiman et Sandra A. Thompson (eds.) Clause combining in grammar and discourse, 135-179.

Lambrecht, K. (1994). Information structure and sentence form: Topic, focus, and the mental representations of discourse referents. Cambridge: Cambridge university press.

Lambrecht, K. (2000). Prédication seconde et structure informationnelle: la relative de perception comme construction présentative. Langue française, 49-66.

Léard, J. M. (1986). Il y a...qui et C'est...qui: La syntaxe comme compatibilité d'opérations sémantiques. Lingvisticae Investigationes, 10(1), 85-130.

Leclercq, P. (2008). L'influence de la langue maternelle chez les apprenants adultes quasibilingues dans une tache contrainte de verbalisation, Acquisition et interaction en langue étrangère, 26, en ligne : http://aile.revues.org/3032

Leclercq, P., et Lenart, E. (2013). Discourse cohesion and accessibility of referents in oral narratives: A comparison of L1 and L2 acquisition of French and English. Discours, (12), en ligne: http://discours.revue.org/8801

Li, C. N. et Thompson, S. A. (1976). Subject and Topic: A New Typology of Language. In Charles N. Li. In Charles Li (Ed.), Subject and Topic, New York: Academic Press. 457-489. 
Li, C. N. et Thompson, S. A. (1981). Mandarin Chinese: a Functional Reference Grammar. Berkeley: University of California Press.

Lü, S. (1942). Zhōngguó wénfă yàolüè [Un aperçu de grammaire chinoise]. Pékin : Presses commerciales.

Paris, M. (1977). Le morphème "de" et la relativation en mandarin. In: Cahiers de linguistique - Asie orientale, (2) 65-76. En ligne: http://www.persee.fr/doc/clao 01533320_1977_num 2_1_1029.

Perdue, C. (1993). Adult language acquisition: Cross-linguistic perspectives. Cambridge: Cambridge University Press.

Perdue, C. (2002). Development of L2 functional use. Portraits of the L2 user. In Vivian Cook (Ed.) Portraits of the L2 user (Vol. 1). Clevedon: Multilingual Matters.

Rickheit, G. et Christopher H. (1995). Focus and Coherence in Discourse Processing. Berlin: Walter de Gruyter.

Ryan, J. (2015). Overexplicit Referent Tracking in L2 English: Strategy, Avoidance, or Myth?. Language Learning, 65(4), 824-859.

Sornicola, R. (2003). Crosslinguistic comparison and second language acquisition: An approach to topic and left detachment constructions from the perspective of spoken language. In Giacalone Ramat A (ed.), Typology and second language acquisition,(pp.7188). Berlin: Mouton de Gruyter.

Sun, J. (2008). Conceptualisation étendue du temps topique dans les narrations des apprenants sinophones en français langue étrangère. Acquisition et interaction en langue étrangère, 26, 71-88.

Tao, H. (1996). Units in Mandarin conversation: Prosody, discourse, and grammar (Vol. 5). Amsterdam : John Benjamins Publishing.

Trévisiol, P., Watorek, M. et Lenart, E. (2010). Topique du discours/topique de l'énoncéréflexions à partir de données en acquisition des langues. In Marina Chini (ed). Topic, information structure and acquisition, (pp. 177-194). Milano : Franco Angeli.

Turco, G. (2008). Introduction et identification d'un référent chez les apprenants francophones de l'italien L2. Acquisition et interaction en langue étrangère, (26), 211-237.

Véronique, G. D. (2013). La dislocation, l'emploi de c'est et il y a et l'organisation topiquefocus en français parlé: des variétés d'apprenants aux usages natifs. TIPA. Travaux interdisciplinaires sur la parole et le langage, 29. En ligne : http://tipa.revues.org/1022

Verwimp, L. et Lahousse, K. (2016). Definite il y a-clefts in spoken French. Journal of French Language Studies, 1- 28. 
Von Stutterheim, C. et Klein, W. (1989). Referential movement in descriptive and narrative discourse. In R. Dietrich et C. Graumann (ed.), Language processing in social context. Amsterdam: North-Holland, 39-76.

Watorek, M., Lenart, E. et Trevisol, P. (2014). The reference to entities in the narrative and descriptive discourses in French L2: the information structure and the use of determination system. Linguistik online, (63), 87-117. 